\title{
ON REGULARIZATION METHODS FOR THE NUMERICAL SOLUTION OF PARABOLIC CONTROL PROBLEMS WITH POINTWISE STATE CONSTRAINTS *
}

\author{
IRA NEITZEL ${ }^{1}$ AND FREDI TRÖLTZSCH ${ }^{1}$
}

\begin{abstract}
In this paper we study Lavrentiev-type regularization concepts for linear-quadratic parabolic control problems with pointwise state constraints. In the first part, we apply classical Lavrentiev regularization to a problem with distributed control, whereas in the second part, a Lavrentiev-type regularization method based on the adjoint operator is applied to boundary control problems with state constraints in the whole domain. The analysis for both classes of control problems is investigated and numerical tests are conducted. Moreover the method is compared with other numerical techniques.
\end{abstract}

Mathematics Subject Classification. 49K20, 49N10, 49M05.

Received January 10, 2007. Revised July 31, 2007 and October 23, 2007.

Published online June 24, 2008.

\section{INTRODUCTION}

In this paper, we consider some aspects of optimal control problems for parabolic equations with pointwise constraints on the state. Our main goal is the investigation of Lavrentiev- and source type regularization methods for parabolic problems. Lavrentiev regularization was introduced for elliptic problems with distributed controls by Meyer et al. in [20], and, in a form closer to this paper, by Meyer and Tröltzsch in [19]. An extension to the case of elliptic boundary control (and pointwise constraints in the domain) was recently suggested in [30]. We discuss the analysis of regularization and its numerical application to distributed and boundary controls. Special emphasis is laid on the case of boundary control.

Let us first motivate our interest in the regularization of state constraints. It is well-known that the Lagrange multipliers associated with these constraints are regular Borel measures, if the states are considered in spaces of continuous functions as in Casas [9] or Raymond and Zidani [25]. The choice of continuous state functions is dictated by Slater type constraint qualifications that need interior points in the cone of nonnegative functions of the space of constraints.

However, this restriction to continuous state functions has some drawbacks. First of all, measures appear in the right-hand sides of associated adjoint equations. In a numerical approximation of the optimal control

\footnotetext{
Keywords and phrases. Optimal control, parabolic equation, pointwise state constraints, boundary control, Lavrentiev-type regularization.

* Research supported by DFG, Priority Program 1253 "Optimierung mit partiellen Differentialgleichungen" ("PDE constrained optimization").

1 Technische Universität Berlin, Fakultät II - Mathematik und Naturwissenschaften, Str. des 17. Juni 136, 10623 Berlin, Germany. neitzel@math.tu-berlin.de; troeltz@math.tu-berlin.de
} 
problems this causes specific difficulties. Second, the control functions must be sufficiently regular to guarantee the continuity of the states. If box constraints are imposed on the control with $L^{\infty}$-bounds, then all controls are bounded and measurable so that continuity of the states is obtained for standard elliptic and parabolic equations in spatial domains of arbitrary finite dimension.

If, however, only state constraints are given, then it is common practice to ensure $L^{2}$-boundedness of the controls by a quadratic Tikhonov regularization term in the objective functional. In this case, one has to work with $L^{2}$-controls, and here the continuity of states is obtained only under severe restrictions on the dimension of the domain and on the type of control: in elliptic problems, distributed controls can be handled up to a dimension of three, while the case of Robin-type boundary control can only be considered up to a dimension of two. The situation is even more difficult for parabolic problems. Here, only distributed controls in onedimensional domains can be admitted. $L^{2}$-Robin-boundary controls in general do not have continuous states.

Both obstacles mentioned above can be avoided by certain regularization techniques. For instance, in the distributed case, Ito and Kunisch [15] introduced a Moreau-Yosida regularization approach method that is based on a penalization of the pointwise state constraints and hereafter permits to apply semismooth Newton methods to solve the resulting unconstrained problems. We refer also to Ito and Kunisch [14], as well as [3-5,17].

Later, in [20,21], a Lavrentiev type regularization was introduced that numerically behaves like the method of $[4,14]$, but, after regularization, preserves the structure of a state-constrained problem. This might be of interest for the convergence analysis of numerical methods in function space. For instance, the convergence of a primal-dual interior point method with regularized pointwise state constraints was shown by Prüfert et al. in [23]. It was recently pointed out by Schiela [29] that the interior point property cannot be expected for purely pointwise state constraints if the standard logarithmic barrier function is applied.

The aim of this paper is to extend Lavrentiev type regularization techniques to parabolic optimal control problems. In Section 2, we will consider problems with distributed control in the whole domain. We will carry out the analysis of a Lavrentiev-regularized problem, placing special emphasis on first order necessary optimality conditions and the convergence analysis for vanishing regularization parameters. Furthermore, numerical experiments are conducted. Section 3 is devoted to boundary control problems, which are also analyzed with respect to optimality conditions and convergence towards the solution of the original unregularized problem. Numerical experiments are conducted for a problem with known analytic solution. We also apply a method by Ito and Kunisch [15] and compare the results. In addition, we consider an approximation to the benchmark problem from [7]. We apply both Lavrentiev-type regularization by a source term representation of the control and the method by Ito and Kunisch, and compare the results to a reference solution obtained by applying the Matlab code quadprog to the unregularized problem.

Overall, we will address three main issues on Lavrentiev-type regularization: First, we will see that the general ideas of Lavrentiev regularization and source term representation from the elliptic case transfer naturally to the parabolic case - as one may expect. Second, and more importantly, we will demonstrate the limits of the analysis of parabolic state-constrained control problems and present a class of problems where analysis is possible. We replace problems where a Lagrange multiplier rule of Karush-Kuhn-Tucker type cannot be established, such as parabolic boundary control problems, or distributed control problems in spatial domains of dimension two or higher, by well-posed problems with existing KKT systems for arbitrary spatial dimensions that in addition preserve the structure of state constraints. The convergence analysis for vanishing Lavrentiev parameters relies on additional assumptions and gives reason to consider a different class of control problems involving state and control constraints in future research. We will argue that the analysis is still applicable to a wide range of problems. Third, we will discuss the well known benchmark problem by Betts and Campbell from $[6,7]$ that originally motivated our research on parabolic boundary control problems, and, as a first step, the work in [30] on elliptic boundary control problems, and suggest a framework for its analysis. We will use an approximation to this model problem, which has also been investigated in the context of direct transcription methods by Kameswaran and Biegler [16] or Betts et al. in [8], for our numerical experiments. 
Let us briefly outline the type of problems we shall investigate. In the distributed case, we consider the problem

$$
\min J(y, u)=\frac{1}{2} \int_{\Omega} \int_{0}^{T}\left\{\left(y(x, t)-y_{Q}(x, t)\right)^{2}+\nu\left(u(x, t)-u_{d}(x, t)\right)^{2}\right\} \mathrm{d} x \mathrm{~d} t
$$

subject to the heat equation

$$
\begin{array}{rll}
\frac{\partial y}{\partial t}-\Delta y=\beta u & & \text { in } Q:=\Omega \times(0, T) \\
\frac{\partial y}{\partial n}+\alpha y=0 & & \text { in } \Sigma:=\Gamma \times(0, T) \\
y(\cdot, 0)=0 & & \text { in } \Omega,
\end{array}
$$

where $n$ denotes the outward unit normal. If only pointwise constraints on the state are given, say

$$
y_{a}(x, t) \leq y(x, t) \leq y_{b}(x, t) \quad \text { in } Q
$$

with fixed $L^{2}$-functions $y_{a}, y_{b}$ such that the admissible set is not empty, then it is easy to show the existence of an optimal control $\bar{u} \in L^{2}(Q)$ with state $\bar{y} \in W(0, T)$. To have a Lagrange multiplier rule of Karush-Kuhn-Tucker type, we need the continuity of $y$ to satisfy a Slater condition. In this case, we require continuity of $y_{a}, y_{b}$, which is not a severe restriction, but we also must restrict the problem to a one-dimensional spatial domain $\Omega$. Even then, the numerical solution of the problem is not easy. Both difficulties can be weakened by Lavrentiev-type regularization: We consider the regularized constraints

$$
y_{a}(x, t) \leq \lambda u(x, t)+y(x, t) \leq y_{b}(x, t) \quad \text { a.e. in } Q
$$

with some small parameter $\lambda \neq 0$. This modification changes the problem, since the constraints are somehow relaxed. However, neither do we need a constraint qualification nor a restriction on the dimension of $\Omega$ to obtain Lagrange multipliers. Moreover, and this is even more important, the Lagrange multipliers are functions in $L^{2}(Q)$, so that measures can be avoided and the optimality system can be solved using standard discretization schemes. We point out, though, that efficient discretization methods for measures have been used in the past, $c f$. for example [11]. Nevertheless, certain results of numerical analysis, such as mesh independence and convergence of interior point methods are not applicable to problems that admit measures as multipliers.

From the viewpoint of optimality conditions, the situation is easier if pointwise constraints on the control are given. Then the restriction of the dimension of $\Omega$ is not needed. Nevertheless, the Lavrentiev-type regularization is helpful. Consider for example nonlinear control problems, where second order sufficient optimality conditions (SSC) are of interest. For unregularized parabolic semilinear problems, SSC have only been proven in full generality for one-dimensional distributed control, cf. [24]. However, in the spirit of [27] a generalization to arbitrary dimensions seems promising for parabolic Lavrentiev regularized problems. We shall investigate distributed problems in Section 2, where we focus mainly on problems without control constraints.

The situation is more complicated for boundary control problems such as

$$
\min J(y, u)=\frac{1}{2} \int_{\Omega} \int_{0}^{T}\left(y(x, t)-y_{Q}(x, t)\right)^{2} \mathrm{~d} x \mathrm{~d} t+\frac{\nu}{2} \int_{\Gamma} \int_{0}^{T}\left(u(x, t)-u_{d}(x, t)\right)^{2} \mathrm{~d} x \mathrm{~d} t
$$

subject to

$$
\begin{aligned}
\frac{\partial y}{\partial t}-\Delta y & =0 & & \text { in } Q:=\Omega \times(0, T) \\
\frac{\partial y}{\partial n}+\alpha y & =\beta u & & \text { in } \Sigma:=\Gamma \times(0, T) \\
y(\cdot, 0) & =0 & & \text { in } \Omega .
\end{aligned}
$$

Consider first the case where only the pointwise constraints on the state (1.3) are given. 
The control-to-state mapping $G: u \mapsto y$ is continuous from $L^{2}(\Sigma)$ to $W(0, T)$, but not from $L^{2}(\Sigma)$ to $C(\bar{Q})$. Therefore, even though the existence of an optimal control is easy to show, we are not able to prove a practicable Lagrange multiplier rule in spaces of $L^{2}$-type. If box constraints on the control are given such that all controls belong to $L^{\infty}(\Sigma)$, then this problem does not appear. But, in view of SSC, it has so far not been possible to show second order sufficient conditions, even for one-dimensional spatial domains. In many papers only state constraints are considered, because the coupling of control and state constraints causes specific numerical problems. Moreover, there arise problems concerning the existence of regular Lagrange multipliers if bilateral box constraints are given for control and state. Hence, there is a special interest in problems with pure state constraints.

A direct application of the Lavrentiev regularization idea to boundary control problems is not obvious. We cannot, as in (1.5), consider the expression $\lambda u+y$ in a meaningful way, since $u$ is defined on $\Sigma$, while $y$ is defined on $Q$. Our main idea to resolve this difficulty is the following: consider the control-to-state mapping $G$ with range in $L^{2}(Q)$ and denote this continuous mapping by $S$. Its adjoint mapping $S^{*}$ maps $L^{2}(Q)$ into $L^{2}(\Sigma)$. Assuming for the moment that $u_{d}=0$, we introduce an auxiliary control $v \in L^{2}(Q)$ and use for $u$ the ansatz $u=S^{*} v$. From the optimality conditions, we expect $\bar{u}=G^{*} \mu$ for the optimal control $\bar{u}$ with some measure $\mu$. By the ansatz $u=S^{*} v$, we perform some smoothing, since we restrict $G^{*}$ from $C(\bar{Q})^{*}$ to $L^{2}(Q)$. Then the state $y=y(v)$ is given by $y=S S^{*} v$ and the state constraints read $y_{a} \leq S S^{*} v \leq y_{b}$. Now, we are able to apply the Lavrentiev-type regularization to these constraints, i.e. we consider

$$
y_{a} \leq \lambda v+y(v) \leq y_{b}
$$

This idea turned out to be useful in the elliptic case, cf. [30], and our experience with parabolic problems is encouraging as well. We shall discuss the consequences, the analysis and the numerical application of this method in Section 3, where we also introduce some minor modification of this idea for an arbitrary $u_{d}$.

In this paper, unless noted otherwise, we agree on the following notation and general assumptions.

Assumption 1.1. The set $\Omega \subset \mathbb{R}^{N}, N \in \mathbb{N}, N \geq 1$, is a bounded open domain with $C^{1,1}$-boundary $\Gamma$; $Q=\Omega \times(0, T), \Sigma=\Gamma \times(0, T)$, with $T \in \mathbb{R}^{+}$. Further, we have positive real numbers $\nu, \lambda$ and $\varepsilon$, and functions $\alpha$ in $L^{\infty}(\Sigma), y_{d} \in L^{2}(Q)$ and bounds $y_{a}, y_{b} \in C(\bar{Q}), y_{a}<y_{b}$, with

$$
y_{a}(x, 0)<0<y_{b}(x, 0) \quad \forall x \in \bar{\Omega} .
$$

If we set $D:=Q$ in the case of distributed control and $D:=\Sigma$ when considering boundary control, the control $u$ and shift control $u_{d}$ are functions in $L^{2}(D)$, while $\beta$ is a function in $L^{\infty}(D)$. We further agree on the short notation $y_{t}:=\frac{\partial y}{\partial t}$ and $\partial_{n} y=\frac{\partial y}{\partial n}$, set $V=H^{1}(\Omega)$ and define the solution space $W(0, T)$ as the space of all $y \in L^{2}(0, T ; V)$ whose (distributional) derivative $y^{\prime}$ exists in $L^{2}\left(0, T ; V^{\star}\right)$.

Remark 1.2. Note that the $C^{1,1}$-regularity of $\Gamma$ is a strong assumption only necessary for the unregularized control problems and when proving convergence results. In the case of Lavrentiev-regularization, where continuity of the state functions is not required, $C^{0,1}$-regularity is sufficient to formulate first-order necessary conditions of Karush-Kuhn-Tucker type.

Before analyzing specific optimal control problems, let us quote the following result on the regularity of the solutions of the parabolic initial-boundary value problems posed in our paper. We consider the problem

$$
\begin{aligned}
y_{t}-\Delta y & =f & & \text { in } Q \\
y(\cdot, 0) & =y_{0} & & \text { in } \Omega \\
\partial_{n} y+\alpha y & =g & & \text { in } \Sigma .
\end{aligned}
$$

Theorem 1.3. Suppose that $\alpha \in L^{\infty}(\Sigma)$ is given and $\Omega \subset \mathbb{R}^{N}$ is a bounded open domain with $C^{1,1}$-boundary $\Gamma$. Then, for all $f \in L^{2}(Q), y_{0} \in L^{2}(\Omega)$, and $g \in L^{2}(\Sigma)$, the problem (1.8) admits a unique solution $y \in W(0, T)$. 
If $f \in L^{r}(Q), r>\frac{N}{2}+1, y_{0} \in C(\bar{Q})$, and $g \in L^{s}(\Sigma), s>N+1$, we obtain $y \in W(0, T) \cap C(\bar{Q})$. In either case the mapping $\left(f, y_{0}, g\right) \mapsto y$ is continuous in the associated spaces.

The proof can be found, for a more general setting, in Casas [10], or Raymond and Zidani [25].

\section{Distributed optimal CONTRol problems}

\subsection{Distributed control with control and state constraints}

We would first like to consider the distributed optimal control problem to minimize the functional (1.1) subject to the parabolic equation (1.2) with the pointwise state constraints

$$
y_{a}(x, t) \leq y(x, t) \leq y_{b}(x, t) \quad \forall x \in \bar{Q},
$$

and with box constraints on the control

$$
u_{a}(x, t) \leq u(x, t) \leq u_{b}(x, t) \quad \text { a.e. in } Q,
$$

where $u_{a}, u_{b}$ are given functions in $L^{\infty}(Q)$.

Applying Theorem 1.3, the presence of box constraints $u_{a}, u_{b} \in L^{\infty}(\Sigma)$ ensures continuity of the state function $y$ and the state inequality constraints can be considered in $C(\bar{Q})$ space. The Lagrange multipliers can be expected to exist in the associated dual space, i.e. in the space $M(\bar{Q})$ of regular Borel measures on $\bar{Q}$. Associated optimality conditions in form of the Pontryagin maximum principle have been derived by Casas [9] and Raymond and Zidani [25]. The following theorem follows immediately from the Pontryagin principle in [9]:

Theorem 2.1. Let $(\bar{u}, \bar{y}) \in L^{2}(Q) \times W(0, T) \cap C(\bar{Q})$ be an optimal solution of the distributed optimal control problem (1.1)-(1.2), (2.1)-(2.2). Assume that $\tilde{u} \in L^{\infty}(Q)$ satisfying (2.2) exists such that the associated state $\tilde{y}$ fulfills (2.1) strictly for all $(x, t) \in \bar{Q}$, i.e. $y_{a}(x, t)+\delta \leq \tilde{y}(x, t) \leq y_{b}(x, t)-\delta$ holds for some $\delta>0$ (Slater condition). Then there exist regular Borel measures $\mu_{a}, \mu_{b} \in M(\bar{Q})$ and an adjoint state $p \in L^{\rho}\left(0, T ; W^{1, \sigma}(\Omega)\right)$, for all $\rho, \sigma \in[1,2)$ with $\frac{2}{\rho}+\frac{N}{\sigma}>N+1$, such that the adjoint equation (2.3), the variational inequality (2.4) and the complementary slackness condition (2.5) hold true:

$$
\begin{aligned}
-p_{t}-\Delta p & =\bar{y}-y_{d}+\mu_{b_{Q}}-\mu_{a_{Q}} \\
p(\cdot, T) & =\mu_{b_{T}}-\mu_{a_{T}} \\
\partial_{n} p+\alpha p & =\mu_{b_{\Sigma}}-\mu_{a_{\Sigma}}, \\
\left(\nu\left(\bar{u}(x, t)-u_{d}(x, t)\right)+\beta p(x, t), v\right. & -\bar{u}) \geq 0 \forall v \in\left[u_{a}(x, t), u_{b}(x, t)\right] \text { a.e. } \quad \text { in } Q, \\
\int_{\bar{Q}}\left(y_{a}-\bar{y}\right) \mathrm{d} \mu_{a}=0, \quad \mu_{a} & \geq 0, \quad \int_{\bar{Q}}\left(\bar{y}-y_{b}\right) \mathrm{d} \mu_{b}=0, \quad \mu_{b} \geq 0,
\end{aligned}
$$

where $\mu_{i_{Q}}=\mu_{i_{\mid Q}}, \mu_{i_{T}}=\mu_{i_{\mid \bar{\Omega} \times T}}$ and $\mu_{i_{\Sigma}}=\mu_{i_{\mid}}, i \in\{a, b\}$, denote the restrictions of $\mu_{a}, \mu_{b}$ to the indicated sets.

Note that $\mu_{\left.a\right|_{0}}=\mu_{b_{\left.\right|_{0}}}=0$ follows from assumption (1.7).

In a Lavrentiev type regularization of that problem, the state constraints (2.1) are replaced by the mixed control-state constraints (1.4). In this situation, more regular Lagrange multipliers $\mu_{a}, \mu_{b} \in L^{2}(Q)$ can be expected, provided that a certain separation condition is satisfied, $c f$. Arada and Raymond [1] or Rösch and Tröltzsch [28]. Roughly speaking, this condition means that lower and upper constraints for the optimal control and state cannot be active at the same time. This assumption depends on the unknown optimal quantities and on $\lambda$. Therefore, it causes some specific difficulties in the analysis for $\lambda \rightarrow 0$. We refer to [22] for further details. 
In case of a unilateral state constraint, the situation is easier. For instance, if the constraint $y_{a} \leq y$ is regularized by

$$
y_{a} \leq \lambda u+y
$$

with $\lambda \neq 0$, then it can be expected that the associated Lagrange multiplier $\mu_{a}$ exists in $L^{2}(Q)$, without any further assumption on the optimal controls. This follows for parabolic equations in the same way as shown by Rösch and Tröltzsch [26], Section 7, for elliptic problems.

Based on these observations, in [20] the constraints $y \geq y_{a}, u \geq 0$ have been considered by their regularization $y+\lambda u \geq y_{a}, u \geq 0$. It was shown in [20], that the associated regularized optimal controls $\bar{u}_{\lambda}$ tend to $\bar{u}$ in $L^{2}(\Omega)$ as $\lambda \rightarrow 0$. The proof can directly be extended to the parabolic case with constraints

$$
\lambda u(x, t)+y(x, t) \geq y_{a}(x, t), \quad u(x, t) \geq 0 .
$$

For this type of constraints, we get $\bar{u}_{\lambda} \rightarrow \bar{u}$ in $L^{2}(Q)$. However, as mentioned above, there are some unsolved problems associated with the constraints $u_{a} \leq u \leq u_{b}$ and $y_{a} \leq \lambda u+y \leq y_{b}$.

\subsection{Distributed control with pure state constraints}

In the case without the box-constraints (2.2) on the controls, continuity of the state functions is not easily obtained and the existence of Lagrange multipliers associated with an optimal solution cannot be proven in general. A loophole is given in the case $N=1$. For $N=1$ and $r=2$ the assumptions of Theorem 1.3 are satisfied with $f=u, g=0$, and $y_{0}=0$. Therefore, the state $y$ is continuous, and the existence of Lagrange multipliers $\mu_{a}, \mu_{b} \in M(\bar{Q})$ for the distributed optimal control problem (1.1)-(1.3), in the following referred to as $(P D)$, can therefore be shown for controls in $L^{2}(Q)$. In this case, Theorem 2.1 needs only a minor modification: the variational inequality (2.4) must be replaced by

$$
\nu\left(\bar{u}-u_{d}\right)+\beta p=0 .
$$

The situation is different if we consider the Lavrentiev-regularized state-constraints

$$
y_{a} \leq \lambda u+y \leq y_{b} \quad \text { a.e. in } Q .
$$

Following [19], the existence of regular Lagrange multipliers in $L^{2}(Q)$ for arbitrary dimension of $\Omega$ is easily shown using a simple substitution technique. We introduce in (2.6) a new control $w:=\lambda u+y=\lambda u+S u$, which yields a purely control-constrained optimal control problem with

$$
y_{a} \leq w \leq y_{b} .
$$

In fact, from $w=\lambda u+y$ we obtain $u=\frac{1}{\lambda}(w-y)$ so that, after inserting this expression for $u$ in equation (1.2), the new equation

$$
y_{t}-\Delta y+\frac{1}{\lambda} y=\frac{\beta}{\lambda} w
$$

is derived along with the control constraints (2.7) and the transformed objective functional

$$
\tilde{J}(y, w)=\frac{1}{2}\left\|y-y_{d}\right\|_{L^{2}(Q)}^{2}+\frac{\nu}{2 \lambda^{2}}\left\|w-y-\lambda u_{d}\right\|_{L^{2}(Q)}^{2} .
$$

The proof of existence of Lagrange multipliers $\mu_{a}, \mu_{b} \in L^{2}(Q)$ for this problem is standard. It can be shown that they are also Lagrange multipliers for the regularized constraints (2.6), cf. [19], i.e. there exist regular Lagrange multipliers for the control problem

$$
\min \left(P D_{\lambda}\right) \quad J(y, u):=\frac{1}{2}\left\|y-y_{d}\right\|_{L^{2}(Q)}^{2}+\frac{\nu}{2}\left\|u-u_{d}\right\|_{L^{2}(Q)}^{2}
$$

subject to the constraints $y_{a} \leq y \leq y_{b}$. The optimality conditions are summarized in the following theorem. 
Theorem 2.2. For each $\lambda>0,\left(P D_{\lambda}\right)$ admits a unique optimal control $\bar{u}$ with associated state $\bar{y}$. For arbitrary $N$, there exist Lagrange multipliers $\mu_{a}, \mu_{b} \in L^{2}(Q)$ and an adjoint state $p \in W(0, T)$ such that:

$$
\begin{aligned}
& -p_{t}-\Delta p=\bar{y}-y_{d}+\mu_{b}-\mu_{a} \\
& p(\cdot, T)=0 \\
& \partial_{n} p+\alpha p=0 \\
& \nu\left(\bar{u}-u_{d}\right)+\beta p+\lambda\left(\mu_{b}-\mu_{a}\right)=0 \\
& \left(\mu_{a}, y_{a}-\lambda \bar{v}-\bar{y}\right)_{L^{2}(Q)}=0 \quad \mu_{a} \geq 0 \quad \text { a.e. in } Q \\
& \left(\mu_{b}, \lambda \bar{v}+\bar{y}-y_{b}\right)_{L^{2}(Q)}=0 \quad \mu_{b} \geq 0 \quad \text { a.e. in } Q \text {. }
\end{aligned}
$$

Remark 2.3. In the sequel, we will replace the objective function $J$ by the reduced objective function $f(u):=$ $J(S u, u)$.

Remark 2.4. We point out that the optimal values of primal and dual variables of the regularized problem, i.e. for example $\bar{u}$, depend on the choice of $\lambda$. However, to keep the notation as simple as possible, we do not express this dependence explicitly.

Remark 2.5. During the following analysis of problem $\left(P D_{\lambda}\right)$ we assume, for simplicity, $u_{d}=0$. Note that this is not a severe restriction, as is explained in more detail for the boundary control case in Section 3.

The principal ideas of the proof of Theorem 2.2 are also explained in Section 3, where we consider a boundary control problem and also transform the problem into a purely control-constrained optimal control problem. Instead of recalling the proof, we focus on convergence issues of the optimal solution $\bar{u}_{\lambda}$ for vanishing Lavrentiev parameter. We recall that, with the assumption $N=1$, the solution operator $G$ maps from $L^{2}(Q)$ to $W(0, T) \cap$ $C(\bar{Q})$. In this case, we are able to show strong convergence of control and state as $\lambda \rightarrow 0$, if we rely on the following assumption.

Assumption 2.6. We assume that there exists a control $u_{0} \in C(\bar{Q})$ such that the Slater condition

$$
y_{a}(x, t)+\delta \leq \bar{y}(x, t)+y_{0}(x, t) \leq y_{b}(x, t)-\delta,
$$

with $\bar{y}=G \bar{u}, y_{0}=G u_{0}$, holds for a given $\delta>0$.

Note that from the existence of $u_{0}$, we find that there exist $\bar{\lambda}>0$ and a control $\hat{u} \in L^{2}(Q)$ feasible for all $\lambda<\bar{\lambda}$. We refer to the proof of Theorem 2.8, where such a control will be constructed. Let now $\left\{\lambda_{n}\right\}$ be a sequence of positive real numbers converging to zero as $n \rightarrow \infty$, and let $\left\{u_{n}\right\}$ denote the sequence of the associated optimal solutions of $\left(P D_{\lambda_{n}}\right)$. With $\nu>0$, we know that

$$
\left\|u_{n}\right\|_{L^{2}(Q)}^{2} \leq \frac{2}{\nu} f(\hat{u}),
$$

since $\hat{u}$ is feasible for $\lambda$ sufficiently small. Thus, $\left\{u_{n}\right\}$ is uniformly bounded in $L^{2}(Q)$, hence there exists a weakly in $L^{2}(Q)$ converging subsequence. W.l.o.g. let $u_{n} \rightarrow u^{\star}, n \rightarrow \infty$.

Lemma 2.7. The weak limit $u^{\star}$ of $\left\{u_{n}\right\}$ is feasible for $(P D)$.

Proof. Since $\left\{u_{n}\right\}$ is uniformly bounded in $L^{2}(Q)$ and $\left\{\lambda_{n}\right\}$ tends to zero, we can assume w.l.o.g. that $\lambda_{n} u_{n} \rightarrow 0$ as $n \rightarrow \infty$. Further, from the compactness of $S: L^{2}(Q) \rightarrow W(0, T) \hookrightarrow L^{2}(Q)$, and the weak convergence of $\left\{u_{n}\right\}$ we obtain

$$
S u_{n} \rightarrow S u^{\star} \text { in } L^{2}(Q) .
$$


We know that $u_{n}$ is feasible for $\left(P D_{\lambda_{n}}\right)$,

$$
y_{a} \leq \lambda_{n} u_{n}+S u_{n} \leq y_{b}
$$

thus, after passing to the limit, due to the fact that $\left[y_{a}, y_{b}\right] \subset L^{2}(Q)$ is closed, we obtain $y_{a} \leq S u^{\star} \leq y_{b}$, i.e. the feasibility of $u^{\star}$ for the unregularized problem $(P D)$.

Theorem 2.8. Let $\left\{\lambda_{n}\right\}$ be a sequence of positive real numbers converging to zero and denote by $\left\{u_{n}\right\}$ the sequence of associated optimal solutions of $\left(P D_{\lambda_{n}}\right)$. For $N=1$, the sequence $\left\{u_{n}\right\}$ converges strongly in $L^{2}(Q)$ towards $\bar{u}$, where $\bar{u}$ is the unique optimal solution of the unregularized problem $(P D)$.

The proof is completely analogous to the one for elliptic problems in [13]. We recall it for convenience of the reader.

Proof. We know that $\bar{u}$ is a function in $L^{2}(Q)$, thus, for $N=1, \bar{y}$ is a continuous function. Further, $C(\bar{Q})$ is dense in $L^{2}(Q)$, hence there exists a sequence $\left\{u_{k}\right\}$ in $C(\bar{Q})$ with

$$
\left\|u_{k}-\bar{u}\right\|_{L^{2}(Q)} \leq \frac{1}{k}
$$

i.e. $\left\{u_{k}\right\}$ converges strongly towards $\bar{u}$ in $L^{2}(Q)$. Since $G: L^{2}(Q) \rightarrow C(\bar{Q})$ is linear and continuous, there exists $c>0$ such that

$$
\left\|G\left(u_{k}-\bar{u}\right)\right\|_{C(\bar{Q})} \leq c\left\|u_{k}-\bar{u}\right\|_{L^{2}(Q)} \leq \frac{c}{k}
$$

With the help of the Slater point $u_{0}$, we define a sequence $\left\{u_{k}^{0}\right\}$ in $C(\bar{Q})$ by

$$
u_{k}^{0}:=u_{k}+\frac{2 c}{\delta k} u_{0}
$$

and obtain

$$
\left\|u_{k}^{0}-\bar{u}\right\|_{L^{2}(Q)} \leq\left\|u_{k}-\bar{u}\right\|_{L^{2}(Q)}+\frac{2 c}{\delta k}\left\|u_{0}\right\|_{C(\bar{Q})} \leq \frac{1}{k}\left(1+\frac{2 c}{\delta}\left\|u_{0}\right\|_{C(\bar{Q})}\right) .
$$

Passing to the limit yields

$$
\lim _{k \rightarrow 0}\left\|u_{k}^{0}-\bar{u}\right\|_{L^{2}(Q)}=0 .
$$

It is now easy to prove that, for $k$ large enough, there exists $n_{k} \in \mathbb{N}$ such that $u_{k}^{0}$ is feasible for all $\left(P D_{\lambda_{n}}\right)$ with $n \geq n_{k}$. We refer to [13] for further details. Note that this proves the existence of the control $u_{k}^{0}$ used to show the uniform boundedness of $\left\{u_{n}\right\}$. Since $u_{n}$ is optimal for $\left(P D_{\lambda_{n}}\right)$, we have

$$
f\left(u_{n}\right) \leq f\left(u_{k}^{0}\right) \quad \forall n \geq n_{k} .
$$

By the lower semi-continuity of $f$ we obtain for each $k$

$$
f\left(u^{\star}\right) \leq \lim _{n \rightarrow \infty} \inf f\left(u_{n}\right) \leq \lim _{n \rightarrow \infty} \sup f\left(u_{n}\right) \leq f\left(u_{k}^{0}\right)
$$

For $k \rightarrow \infty$ we arrive at

$$
f\left(u^{\star}\right) \leq \lim _{k \rightarrow \infty} f\left(u_{k}^{0}\right)=f(\bar{u}) \leq f\left(u^{\star}\right),
$$

hence $f\left(u^{*}\right)=f(\bar{u})$. By the uniqueness of $\bar{u}$, we deduce $u^{*}=\bar{u}$, which implies the optimality of $u^{\star}$ for $(P D)$. It remains to show that the convergence is strong in $L^{2}(Q)$. From the above, we know that

$$
\lim _{n \rightarrow \infty} f\left(u_{n}\right)=f(\bar{u}),
$$


and from the compactness of $S$ we further deduce that

$$
\lim _{n \rightarrow \infty}\left\|S u_{n}-y_{d}\right\|_{L^{2}(Q)}^{2}=\left\|S \bar{u}-y_{d}\right\|_{L^{2}(Q)}^{2} .
$$

Conditions (2.10) and (2.11) imply that

$$
\lim _{n \rightarrow \infty}\left\|u_{n}\right\|_{L^{2}(Q)}^{2}=\|\bar{u}\|_{L^{2}(Q)}^{2} .
$$

That, together with the weak convergence, implies strong convergence.

Remark 2.9. If we assume the optimal control to be a function in $L^{r}(Q), r>\frac{N}{2}+1$, the proof can be extended to $N>1$, since in this case $G \bar{u}$ and $G u_{0}$ are elements of $W(0, T) \cap C(\bar{Q})$. This implies in particular that the last theorem guarantees strong convergence for all dimensions for optimal controls $\bar{u}$ belonging to $L^{\infty}(Q)$. Since bounded controls can be observed in many cases even without additional $L^{\infty}$-bounds on the control it seems reasonable to assume that the convergence analysis is applicable if the numerical results indicate that the optimal control is bounded. However, we point out that the restriction on the dimension appears in other regularization approaches as well. We mention the Moreau-Yosida regularization approach by Ito and Kunisch, cf. [15], where an elliptic distributed control problem is considered up to a dimension of 3 . This is also due to the fact that in this setting continuous state functions are obtained. The parabolic analogon to this situation is a space dimension of $N=1$ for distributed controls.

Remark 2.10. Generally, it is possible to consider Lavrentiev parameters $\lambda<0$. When introducing the control $w=(\lambda I+S) v$ the key idea is the existence of the inverse operator $(\lambda I+S)^{-1}$. The theory of Fredholm operators ensures this if $\lambda \neq 0$ is not an eigenvalue of $S$. While for positive definite operators in general this is true for any $\lambda>0$, we can make use of the fact that the parabolic operator $S$ does not have eigenvalues and use any $\lambda \neq 0$ as a regularization parameter. The situation is different for elliptic problems, where $S$ is self-adjoint, hence normal, as well as the regularization approach for parabolic boundary problems, where the self-adjoint operator $S S^{*}$ is considered, $c f$. Section 3.

\subsection{Active set algorithm}

In this section we briefly present a practicable method to solve problem $\left(P D_{\lambda}\right)$. Primal-dual active-set strategies have been studied in detail for example by Bergounioux and Kunisch [3] and Bergounioux et al. [4], or Ito and Kunisch [15]. Based on the experiences with semismooth Newton methods in [13,30] for elliptic distributed and boundary control problems, and the results in [12], where the active set strategy was interpreted as a semismooth Newton method, we present an active set strategy that can also be understood as a semismooth Newton method for linear quadratic control problems. Following [30], we derive the solution algorithm. First, it can be shown that the complementary slackness conditions (2.9) are equivalent to

$$
\begin{aligned}
& \mu_{a}=\max \left(0, \mu_{a}-\mu_{b}+c\left(y_{a}-\lambda \bar{u}-\bar{y}\right)\right) \\
& \mu_{b}=\max \left(0, \mu_{b}-\mu_{a}+c\left(\lambda \bar{u}+\bar{y}-y_{b}\right)\right)
\end{aligned}
$$

for an arbitrarily fixed $c>0$. Choosing $c=\frac{\nu}{\lambda^{2}}$, we easily obtain with the help of equation (2.8) that the complementary slackness conditions are equivalent to

$$
\begin{aligned}
& \mu_{a}=\max \left(0, \frac{\beta}{\lambda} p-\frac{\nu}{\lambda} u_{d}+\frac{\nu}{\lambda^{2}}\left(y_{a}-\bar{y}\right)\right) \\
& \mu_{b}=\max \left(0, \frac{\nu}{\lambda} u_{d}-\frac{\beta}{\lambda} p+\frac{\nu}{\lambda^{2}}\left(\bar{y}-y_{b}\right)\right)
\end{aligned}
$$


With the definition of the active sets $\mathcal{A}_{a}$ and $\mathcal{A}_{b}$ associated with $p$ and $y$,

$$
\begin{aligned}
& \mathcal{A}_{a}(y, p):=\left\{(x, t) \in Q \mid \beta p-\nu u_{d}+\frac{\nu}{\lambda}\left(y_{a}-y\right)>0 \text { a.e. in } Q\right\} \\
& \mathcal{A}_{b}(y, p):=\left\{(x, t) \in Q \mid \nu u_{d}-\beta p+\frac{\nu}{\lambda}\left(y-y_{b}\right)>0 \text { a.e. in } Q\right\}
\end{aligned}
$$

we set up the following solution algorithm

\section{Algorithm 1.}

(1) Initialize $\left(y_{0}, u_{0}, p_{0}, \mu_{a, 0}, \mu_{b, 0}\right)$ and set $n=0$.

(2) Determine the sets $\mathcal{A}_{a, n}=\mathcal{A}_{a}\left(y_{n}, p_{n}\right)$ and $\mathcal{A}_{b, n}=\mathcal{A}_{b}\left(y_{n}, p_{n}\right)$ by (2.12) and (2.13).

(3) Find $\left(y_{n+1}, u_{n+1}, p_{n+1}, \mu_{a_{n+1}}, \mu_{b_{n+1}}\right)$ that solve

$$
(O P T)\left\{\begin{aligned}
y_{n+1} & =S u_{n+1} \\
\beta p_{n+1} & =S^{*}\left(y_{n+1}-y_{d}+\mu_{b_{n+1}}-\mu_{a_{n+1}}\right) \\
0 & =\nu\left(u_{n+1}-u_{d}\right)+\beta p_{n+1}+\lambda\left(\mu_{b_{n+1}}-\mu_{a_{n+1}}\right) \\
\mu_{a_{n+1}} & =\left(\frac{\beta}{\lambda} p_{n+1}-\frac{\nu}{\lambda} u_{d}+\frac{\nu}{\lambda^{2}}\left(y_{a}-y_{n+1}\right)\right)_{\mathcal{X}_{\mathcal{A}_{a, n}}} \\
\mu_{b_{n+1}} & =\left(\frac{\nu}{\lambda} u_{d}-\frac{\beta}{\lambda} p_{n+1}+\frac{\nu}{\lambda^{2}}\left(y_{n+1}-y_{b}\right)\right)_{\mathcal{X}_{\mathcal{A}_{b, n}}}
\end{aligned}\right.
$$

(4) If $n \geq 1$ and $\mathcal{A}_{a, n+1}=\mathcal{A}_{a, n}$ and $\mathcal{A}_{b, n+1}=\mathcal{A}_{b, n}$ STOP, else set $n=n+1$ and go to step (2).

The initial values for the algorithm are determined as the solution of the problem without state constraints, using $\mathcal{A}_{a,-1}=\mathcal{A}_{b,-1}=\emptyset$. In terms of PDEs, solving $(O P T)$ pertains to solving the following linear system

$$
\begin{aligned}
& y_{t}-\Delta y=\beta u \quad-p_{t}-\Delta p=y-y_{d}+\mu_{b}-\mu_{a} \\
& y(\cdot, 0)=0 \quad p(\cdot, T)=0 \\
& \partial_{n} y+\alpha y=0 \quad \partial_{n} p+\alpha p=0 \\
& \nu\left(u-u_{d}\right)+\beta p+\lambda\left(\mu_{b}-\mu_{a}\right)=0 \quad \text { on } \Sigma \\
& \mu_{a}=\left\{\begin{array}{cl}
\frac{\beta}{\lambda} p-\frac{\nu}{\lambda} u_{d}+\frac{\nu}{\lambda^{2}}\left(y_{a}-y\right), & \text { on } \mathcal{A}_{a} \\
0, & \text { on } \mathcal{A}_{b} \cup \mathcal{I}
\end{array}\right. \\
& \mu_{b}=\left\{\begin{array}{cl}
\frac{\nu}{\lambda} u_{d}-\frac{\beta}{\lambda} p+\frac{\nu}{\lambda^{2}}\left(y-y_{b}\right), & \text { on } \mathcal{A}_{b} \\
0, & \text { on } \mathcal{A}_{a} \cup \mathcal{I}
\end{array}\right.
\end{aligned}
$$

for the corresponding iterates, which we solve at once by complete discretization in space and time using the backslash operator in Matlab. This method is acceptable for one-dimensional problems but certainly more sophisticated methods should be considered for higher dimensions.

\subsection{Numerical experiments}

In the following, we test this regularization method on simple model problems. Our goal is to numerically confirm convergence of the solution of $\left(P D_{\lambda}\right)$ towards the true solution of $(P D)$ as $\lambda$ tends to zero. We consider first a problem $\left(P D_{1 D}\right)$ in $Q=(0,1) \times(0,1)$ with known solution and the data $\alpha=0$ and $\beta=1$, inhomogeneous boundary conditions $\partial_{n} y=g$ on $\Sigma$, where $g=-\pi \sin (\pi t)$, and one-sided pointwise state constraints, i.e.

$$
\min \quad J(y, u):=\frac{1}{2}\left\|y-y_{d}\right\|_{L^{2}(Q)}^{2}+\frac{\nu}{2}\left\|u-u_{d}\right\|_{L^{2}(Q)}^{2}
$$


subject to

We define

$$
\begin{aligned}
y_{t}(x, t)-y_{x x}(x, t) & =u(x, t) & & \text { in } Q \\
y(x, 0) & =0 & & \text { in } \Omega \\
-y_{x}(0, t) & =-\pi \sin (\pi t) & & \text { on } \Sigma_{1}=\{0\} \times(0,1) \\
y_{x}(1, t) & =-\pi \sin (\pi t) & & \text { on } \Sigma_{2}=\{1\} \times(0,1) \\
y(x, t) & \leq y_{b}(x, t) & & \text { a.e. in } Q .
\end{aligned}
$$

and specify the data through

$$
\begin{aligned}
y_{d} & =\hat{y}+\max (0, \hat{y}-c)-\cos (\pi x)-(1-t) \pi^{2} \cos (\pi x) \\
u_{d} & =\hat{y}_{t}-\hat{y}_{x x}+\frac{1}{\nu}(1-t) \cos (\pi x) \\
y_{b} & =\max (c, \hat{y})
\end{aligned}
$$

for a real number $0<c<1$ and $\nu>0$. It is easily verified that

$$
\bar{y}=\hat{y}, \quad \bar{u}=\hat{y}_{t}-\hat{y}_{x x}
$$

is an optimal solution with associated adjoint state $p$ and Lagrange multiplier $\mu$, where

$$
p=(1-t) \cos (\pi x), \quad \mu=\max (0, \hat{y}-c) \text {. }
$$

For our numerical experiments we try Algorithm 1 with various $\lambda$. We do not carry out a comprehensive analysis of the dependence between the regularization parameter $\lambda$ and the grid parameters $\tau$ and $h$, since this would be beyond the scope of this paper. Instead, we choose a fixed grid with $n_{x}=101$ equidistant points in the spatial domain $\bar{\Omega}=[0,1]$, and $n_{t}=101$ equidistant points in the time domain $[0,1]$, i.e. with grid parameters $\tau=h=10^{-2}$ and try different values of $\lambda$ to gain an idea about the appropriate range of values on the given grid. We use a standard central difference approximation of the Laplace operator and an implicit Euler scheme for the time integration to solve the differential equations numerically. We verify the quality of the approximation by computing the relative $L^{2}$-error of $\bar{y}_{\tau h}$ and $\bar{u}_{\tau h}$, denoted by dist $t_{y}$ and dist $_{u}$, respectively, for different $\lambda$ and fixed values $c=0.5, \nu=10^{-4}$, on the same grid. Even though we have not studied the convergence of the regularized adjoint state $p_{\lambda}$ and multiplier $\mu_{\lambda}$ towards $p$ and $\mu$ analytically, we include the $L^{2}$-error in our numerical studies. The results are summarized in Table 1 . For essentially bounded $\left\{u_{\lambda}\right\}$, we expect a convergence rate of $\sqrt{\lambda}$ for $\frac{\left\|\bar{u}-\bar{u}_{\lambda}\right\|}{\|\bar{u}\|}$, cf. [23], Section 7. Referring to Figure 5 , we observe for this problem a convergence rate of $\lambda$, as long as $\lambda>10^{-6}$. From the results in Table 1 and Figure 5 , we conclude that a $\lambda$ of approximately $10^{-5}$ seems to be optimal. Figures $1-4$ show the numerical solution of $\left(P D_{1 D}\right)$ for a moderate $\lambda=10^{-4}$, which already yields satisfying results. Note that due to the nature of the model problem the Lagrange multiplier for the unregularized problem is a continuous function instead of a Borel measure. We refer to Section 3.3 for a measure-valued example.

\section{BOUNDARY CONTROL PROBLEMS}

This section is devoted to the study of the boundary control problem $(P B)$ to minimize the functional (1.5), subject to the state equation (1.6) and the state constraints (1.3). It is known that the initial-boundary-value problem (1.6) admits a unique solution $y \in W(0, T)$ for any given control $u \in L^{2}(\Sigma)$. Thus, the proof of existence of a unique solution of problem $(P B)$ is standard if the set of admissible controls is not empty. On the other hand, first-order optimality conditions of Karush-Kuhn-Tucker-type cannot be formulated in useful spaces due to the lack of continuity of the state functions. If we assume that the optimal control $\bar{u}$ is 


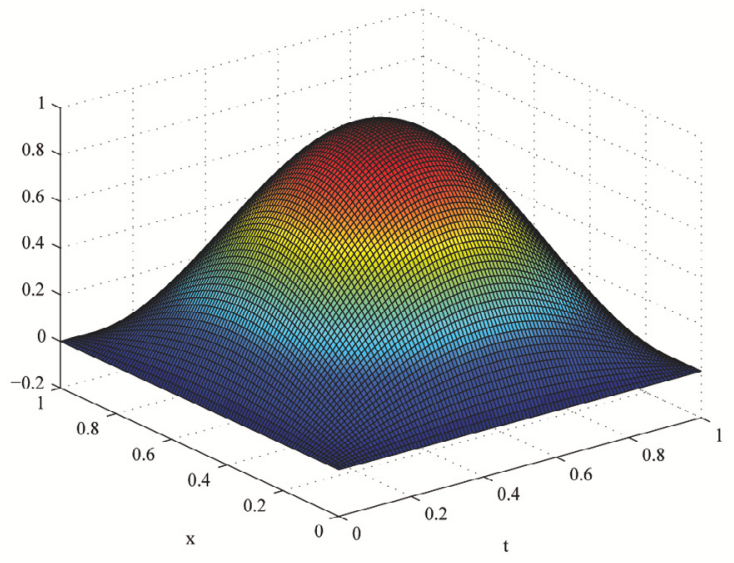

FigURE 1. $\bar{y}_{\tau h}$ at $c=0.5$.

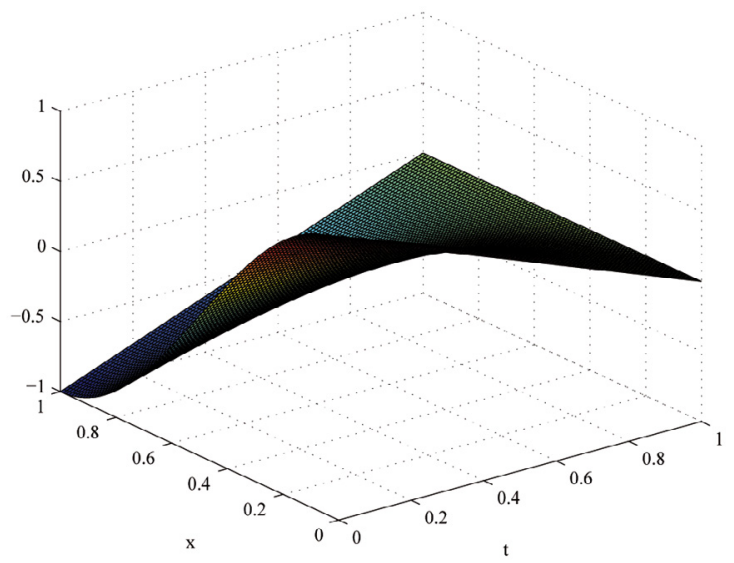

FiguRE $3 . p_{\tau h}$ at $c=0.5$.

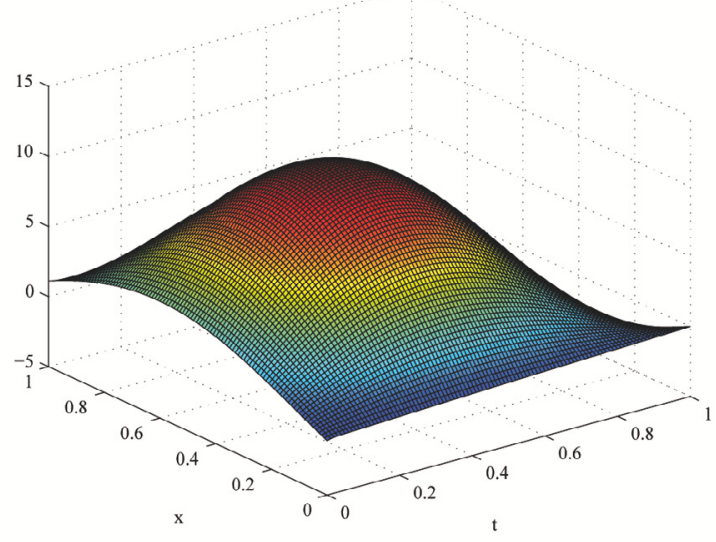

FiguRE 2. $\bar{u}_{\tau h}$ at $c=0.5$.

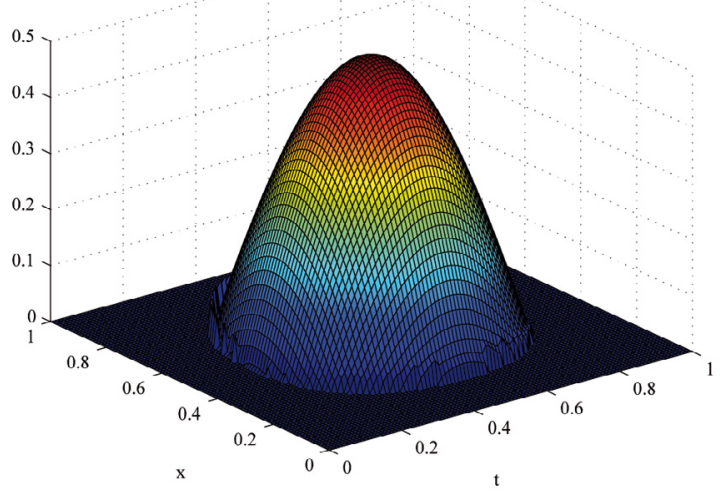

FiguRE $4 . \mu_{\tau h}$ at $c=0.5$.

TABLE 1. Convergence behavior of Algorithm 1 for $\lambda \rightarrow 0$.

\begin{tabular}{|c|c|c|c|c|}
\hline$\lambda$ & dist $_{y}$ & dist $_{u}$ & dist $_{p}$ & dist $_{\mu}$ \\
\hline $10^{0}$ & $3.8983201 \mathrm{e}+00$ & $3.5380126 \mathrm{e}+03$ & $2.1500435 \mathrm{e}+00$ & $4.8092631 \mathrm{e}+00$ \\
\hline $10^{-1}$ & $7.0033702 \mathrm{e}-01$ & $1.2233411 \mathrm{e}+02$ & $1.0482033 \mathrm{e}-01$ & $1.9798384 \mathrm{e}+00$ \\
\hline $10^{-2}$ & $7.9585814 \mathrm{e}-02$ & $8.6762701 \mathrm{e}+00$ & $4.1021905 \mathrm{e}-03$ & $2.1166864 \mathrm{e}-01$ \\
\hline $10^{-3}$ & $9.2490812 \mathrm{e}-03$ & $8.6455326 \mathrm{e}-01$ & $4.0137371 \mathrm{e}-04$ & $8.2024717 \mathrm{e}-02$ \\
\hline $10^{-4}$ & $1.0725655 \mathrm{e}-03$ & $8.8557418 \mathrm{e}-02$ & $4.4965393 \mathrm{e}-05$ & $2.4133410 \mathrm{e}-02$ \\
\hline $10^{-5}$ & $4.7678684 \mathrm{e}-04$ & $1.1836433 \mathrm{e}-02$ & $1.0000534 \mathrm{e}-05$ & $2.5259377 \mathrm{e}-02$ \\
\hline $10^{-6}$ & $4.6704645 \mathrm{e}-04$ & $5.7435933 \mathrm{e}-03$ & $6.9684751 \mathrm{e}-06$ & $2.7761071 \mathrm{e}-02$ \\
\hline $10^{-7}$ & $4.6696175 \mathrm{e}-04$ & $5.3767160 \mathrm{e}-03$ & $6.6950402 \mathrm{e}-06$ & $2.8038172 \mathrm{e}-02$ \\
\hline
\end{tabular}




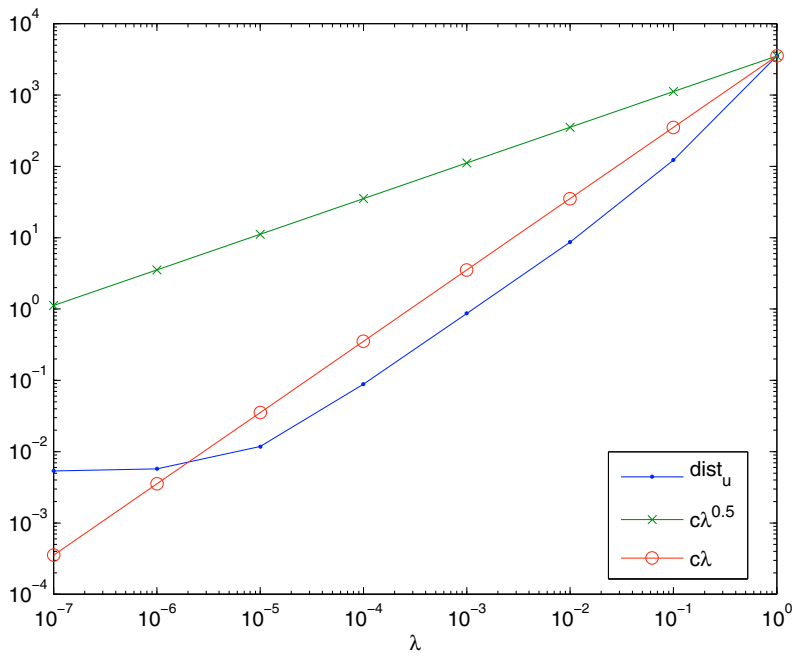

Figure 5. Relative $L^{2}$-error of $\bar{u}_{\tau h}$.

a function in $L^{s}(\Sigma), s>N+1$, we can formally restrict problem $(P B)$ to controls in $L^{s}(\Sigma)$. Note that even for $N=1$ this excludes $L^{2}(\Sigma)$. Then continuity of the state functions is given, and by Theorem 1.3 optimality conditions can be derived using a Slater condition. The existence of such an optimal solution, however, cannot be guaranteed, unless we have constructed test examples where $\bar{y}$ is known to be continuous. Alternatively, one may assume that the optimal control is bounded if the numerical numerical solution is bounded. The situation improves significantly when considering a Lavrentiev-regularized version of $(P B)$. We recall that our main idea of Lavrentiev-regularization for boundary control is the introduction of an auxiliary distributed control $v$ via the source term representation

$$
u=S^{*} v .
$$

It is known that $S^{*} v=\beta z_{\mid \Sigma}$, where $z \in W(0, T)$ solves

$$
\begin{array}{r}
-z_{t}-\Delta z=v \\
z(\cdot, T)=0 \\
\partial_{n} z+\alpha z=0,
\end{array}
$$

$c f .$, for instance [18]. This forces the value $u(x, T)$ to be zero in any case. Hence, we choose to set

$$
u-u_{d}=: \tilde{u}=S^{*} v .
$$

This yields

$$
y=S u=S\left(\tilde{u}+u_{d}\right)=S S^{*} v+S u_{d}
$$

and thus

$$
y_{a} \leq S S^{*} v+S u_{d} \leq y_{b} .
$$

It is clear that the fixed term $u_{d}$ does not influence the optimization process and can be eliminated by redefining $y_{a}:=y_{a}-S u_{d}, y_{b}:=y_{b}-S u_{d}$ and $y_{d}:=y_{d}-S u_{d}$. Therefore, restricting the discussion to problems with $u_{d}=0$ is justified. We study the regularized modified problem $\left(P B_{\lambda}\right)$, where the goal is to minimize the functional $J_{\varepsilon}$

$$
\min J_{\varepsilon}(y, u, v):=\frac{1}{2}\left\|y-y_{d}\right\|_{L^{2}(Q)}^{2}+\frac{\nu}{2}\|u\|_{L^{2}(\Sigma)}^{2}+\frac{\varepsilon(\lambda)}{2}\|v\|_{L^{2}(Q)}^{2},
$$


subject to

$$
y=S u, \quad u=S^{*} v
$$

and the regularized state-control constraints

$$
y_{a}(x, t) \leq \lambda v(x, t)+y(x, t) \leq y_{b}(x, t) \quad \text { a.e. in } Q .
$$

After replacing $u=S^{*} v=\beta z_{\mid \Sigma}$ this yields

$$
\min J_{\varepsilon}(y, z, v):=\frac{1}{2}\left\|y-y_{d}\right\|_{L^{2}(Q)}^{2}+\frac{\nu}{2}\|\beta z\|_{L^{2}(\Sigma)}^{2}+\frac{\varepsilon(\lambda)}{2}\|v\|_{L^{2}(Q)}^{2}
$$

subject to

$$
\begin{array}{rlrl}
y_{t}(x, t)-\Delta y(x, t) & =0 & -z_{t}(x, t)-\Delta z(x, t) & =v(x, t) \\
y(x, 0) & =0 & z(x, T) & =0 \\
\partial_{n} y(x, t)+\alpha y(x, t) & =\beta^{2} z(x, t) & \partial_{n} z(x, t)+\alpha z(x, t) & =0
\end{array}
$$

and the state constraints $y_{a} \leq \lambda v+y \leq y_{b}$.

Note that for a fixed $\lambda$, the problem is solvable for $\varepsilon=0$, and the additional term $\frac{\varepsilon(\lambda)}{2}\|v\|_{L^{2}(Q)}^{2}$ that we introduced in this problem formulation is not necessary. Clearly, $y$ is bounded in $L^{2}(Q)$, hence also $v$ is bounded with values in $\left[\frac{1}{\lambda}\left(y_{a}-y\right), \frac{1}{\lambda}\left(y_{b}-y\right)\right]$. However, we need $\varepsilon>0$ to show a convergence result for $\lambda$ tending to zero, as well as for the uniqueness of $\bar{v}$. We will show later that there exists a dependence between $\varepsilon$ and $\lambda$ to guarantee convergence and have therefore denoted this relationship by $\varepsilon(\lambda)$. Consequently, we finally need only one regularization parameter $\lambda$.

\subsection{Optimality conditions and regular Lagrange multipliers}

To derive the necessary optimality conditions for $\left(P B_{\lambda}\right)$ we introduce the reduced objective function $f_{\varepsilon}$,

$$
J_{\varepsilon}(y, u, v)=J_{\varepsilon}\left(S S^{*} v, S^{*} v, v\right)=: f_{\varepsilon}(v)
$$

and find that $\left(P B_{\lambda}\right)$ is equivalent to minimizing $f_{\varepsilon}(v)$ subject to

$$
y_{a}(x, t) \leq\left(\lambda v+S S^{*} v\right)(x, t) \leq y_{b}(x, t) \quad \text { a.e. in } \mathrm{Q} .
$$

Here and in the following, we drop the dependence of $\varepsilon$ on $\lambda$. To analyze problem $\left(P B_{\lambda}\right)$ in this form, we substitute $\lambda v+S S^{*} v=w$ and consider the linear equation

$$
\left(\lambda I+S S^{*}\right) v=w,
$$

where $I$ denotes the identity operator. Since $S S^{*}$ is positive definite and compact in $L^{2}(Q),\left(\lambda I+S S^{*}\right)$ has, for every $\lambda>0$, an inverse operator $K: L^{2}(Q) \rightarrow L^{2}(Q)$,

$$
K=\left(\lambda I+S S^{*}\right)^{-1}
$$

due to the Fredholm alternative. Thus, we obtain with $v=K w$

$$
f_{\varepsilon}(v)=f_{\varepsilon}(K w)=: F(w) .
$$

In this way, we have transformed problem $\left(P B_{\lambda}\right)$ into the purely control-constrained optimal control problem

$$
(P W) \begin{cases}\text { minimize } & F(w) \\ \text { subject to } & w \in W_{a d}\end{cases}
$$


where the admissible set $W_{a d}$ is defined by

$$
W_{a d}:=\left\{w \in L^{2}(Q) \mid y_{a}(x, t) \leq w(x, t) \leq y_{b}(x, t) \text { a.e. in } Q\right\} .
$$

In the remainder of this section, we derive first-order optimality conditions, following [20]. Even though the procedures are common practice we explain the principle ideas in detail. Since $J_{\varepsilon}$ is differentiable the same holds for $f_{\varepsilon}$, and thus $F$, since $K$ is a linear and continuous operator. The optimality conditions follow from the well-known variational inequality

$$
F^{\prime}(\bar{w})(w-\bar{w}) \geq 0 \quad \forall w \in W_{a d} .
$$

$F^{\prime}(\bar{w})$ can, by the Riesz-Theorem, be identified with a function of $L^{2}(Q)$. We denote this function by $\mu$ and obtain

$$
F^{\prime}(\bar{w}) w=\int_{Q} \mu(x, t) w(x, t) \mathrm{d} x \mathrm{~d} t .
$$

With splitting of $\mu$ into nonnegative $L^{2}$-functions

$$
\mu_{a}=\mu_{+}=\frac{1}{2}(\mu+|\mu|) \text { and } \mu_{b}=\mu_{-}=\frac{1}{2}(\mu-|\mu|),
$$

we obtain $\mu=\mu_{a}-\mu_{b}$ and

$$
F^{\prime}(\bar{w})+\mu_{b}-\mu_{a}=0 .
$$

In a standard way, the variational inequality (3.4) implies $\mu(x, t)=0$ almost everywhere where $y_{a}(x, t)<$ $\bar{w}(x, t)<y_{b}(x, t)$, and $\bar{w}(x, t)=y_{a}(x, t)$, where $\mu(x, t)>0$ and $\bar{w}(x, t)=y_{b}(x, t)$, where $\mu(x, t)<0$. From this, we immediately deduce the conditions

$$
0=\int_{Q} \mu_{a}(x, t)\left(\bar{w}(x, t)-y_{a}(x, t)\right) \mathrm{d} t=\int_{Q} \mu_{b}(x, t)\left(\bar{w}(x, t)-y_{b}(x, t)\right) \mathrm{d} t .
$$

In the following, we will show that the functions $\mu_{a}, \mu_{b} \in L^{2}(Q)$ are Lagrange multipliers for the control-state constraints (3.2).

Theorem 3.1. Let $\bar{v} \in L^{2}(Q)$ be the optimal control for $\left(P B_{\lambda}\right)$ with associated states $\bar{y}$ and $\bar{z}$ and boundary control $\bar{u}$. Then there exist Lagrange multipliers $\mu_{a}, \mu_{b} \in L^{2}(Q)$ and adjoint states $p, q \in W(0, T)$ such that:

$$
\begin{aligned}
-p_{t}-\Delta p & =\bar{y}-y_{d}+\mu_{b}-\mu_{a} \\
p(\cdot, T) & =0 \\
\partial_{n} p+\alpha p & =0
\end{aligned}
$$

$$
\begin{aligned}
q_{t}-\Delta q & =0 \\
q(\cdot, 0) & =0 \\
\partial_{n} q+\alpha q & =\beta^{2}(\nu z+p)
\end{aligned}
$$

$$
\begin{array}{ccc}
\left(\mu_{a}, y_{a}-\lambda \bar{v}-\bar{y}\right)_{L^{2}(Q)}=0, & \mu_{a} \geq 0 \\
\left(\mu_{b}, \lambda \bar{v}+\bar{y}-y_{b}\right)_{L^{2}(Q)}=0, & \mu_{b} \geq 0 \\
\varepsilon \bar{v}+q+\lambda\left(\mu_{b}-\mu_{a}\right)=0 . &
\end{array}
$$

Proof. We first express $F^{\prime}$ in terms of $f_{\varepsilon}$ and $\bar{v}$. With $F(\bar{w})=f_{\varepsilon}(K \bar{w}), K$ defined by (3.3), using the chain rule yields

$$
F^{\prime}(\bar{w}) w=f_{\varepsilon}^{\prime}(K \bar{w}) K^{\prime}(\bar{w}) w=f_{\varepsilon}^{\prime}(K \bar{w}) K w .
$$

This makes equation (3.5) equivalent to

$$
f_{\varepsilon}^{\prime}(K \bar{w}) K w+\left(\mu_{b}-\mu_{a}, w\right)_{L^{2}(Q)}=0 \quad \forall w \in L^{2}(Q) .
$$


If we now substitute $v=K w, \bar{v}=K \bar{w}$, we obtain

$$
f_{\varepsilon}^{\prime}(\bar{v}) v+\left(\mu_{b}-\mu_{a},\left(\lambda I+S S^{*}\right) v\right)_{L^{2}(Q)}=0 .
$$

We know that

$$
\begin{aligned}
f_{\varepsilon}^{\prime}(\bar{v}) v & =\left(S S^{*} \bar{v}-y_{d}, S S^{*} v\right)_{L^{2}(Q)}+\nu\left(S^{*} \bar{v}, S^{*} v\right)_{L^{2}(\Sigma)}+\varepsilon(\bar{v}, v)_{L^{2}(Q)} \\
& =\left(S S^{*}\left(\bar{y}-y_{d}\right)+\nu S S^{*} \bar{v}+\varepsilon \bar{v}, v\right)_{L^{2}(Q)} \\
& =\left(S \beta p_{1}+\nu S \beta z+\varepsilon \bar{v}, v\right)_{L^{2}(Q)} \\
& =\left(S\left(\beta p_{1}+\nu \beta z\right)+\varepsilon \bar{v}, v\right)_{L^{2}(Q)} \\
& =\left(q_{1}+\varepsilon \bar{v}, v\right)_{L^{2}(Q)},
\end{aligned}
$$

where $z=S^{*} \bar{v}$, and $p_{1}, q_{1}$ are the weak solutions of

$$
\begin{aligned}
-p_{1_{t}}-\Delta p_{1} & =\bar{y}-y_{d} & q_{1_{t}}-\Delta q_{1} & =0 \\
p_{1}(\cdot, T) & =0 & q_{1}(\cdot, 0) & =0 \\
\partial_{n} p_{1}+\alpha p_{1} & =0 & \partial_{n} q_{1}+\alpha q_{1} & =\beta^{2}\left(p_{1}+\nu z\right)
\end{aligned}
$$

respectively, according to the definitions of $S$ and $S^{*}$.

The second term in (3.10) can be expressed by

$$
\begin{aligned}
\left(\mu_{b}-\mu_{a},\left(\lambda I+S S^{*}\right) v\right)_{L^{2}(Q)} & =\lambda\left(\mu_{b}-\mu_{a}, v\right)_{L^{2}(Q)}+\left(\mu_{b}-\mu_{a}, S S^{*} v\right)_{L^{2}(Q)} \\
& =\lambda\left(\mu_{b}-\mu_{a}, v\right)_{L^{2}(Q)}+\left(S S^{*}\left(\mu_{b}-\mu_{a}\right), v\right)_{L^{2}(Q)} \\
& =\lambda\left(\mu_{b}-\mu_{a}, v\right)_{L^{2}(Q)}+\left(S \beta p_{2}, v\right)_{L^{2}(Q)} \\
& =\lambda\left(\mu_{b}-\mu_{a}, v\right)_{L^{2}(Q)}+\left(q_{2}, v\right)_{L^{2}(Q)}
\end{aligned}
$$

where $p_{2}=S^{*}\left(\mu_{b}-\mu_{a}\right)$ and $q_{2}=S\left(\beta p_{2}\right)$ solve

$$
\begin{aligned}
& -p_{2_{t}}-\Delta p_{2}=\mu_{b}-\mu_{a} \\
& p_{2}(\cdot, T)=0 \\
& q_{2_{t}}-\Delta q_{2}=0 \\
& \partial_{n} p_{2}+\alpha p_{2}=0 \\
& \begin{aligned}
q_{2}(\cdot, 0) & =0 \\
\partial_{n} q_{2}+\alpha q_{2} & =\beta^{2} p_{2} .
\end{aligned}
\end{aligned}
$$

The adjoint variables $p_{1}, p_{2}$ and $q_{1}, q_{2}$ are functions in $W(0, T)$. It is clear that $p:=p_{1}+p_{2}$ and $q:=q_{1}+q_{2}$ solve the adjoint systems (3.7) and (3.8), respectively, and the gradient equation (3.9) is fulfilled.

The conditions (3.9) follow immediately from (3.6) and the definition of $\mu_{a}, \mu_{b}$.

\subsection{Pass to the limit}

As in the case of distributed control, we are interested in convergence of the regularized solution when the Lavrentiev parameter tends to zero. With the assumption of continuity of the state, i.e. an optimal control in $L^{s}(\Sigma), s>N+1$, or even bounded optimal controls in $L^{\infty}(\Sigma)$, the proof is analogous to the convergence proof for regularized elliptic boundary control problems in [30]. The principal idea is the same as in the distributed case. We rely on the following assumptions: 
Assumption 3.2. Let $\bar{u}$ belong to $L^{s}(\Sigma), s>N+1$, and let there exists a Slater point $v_{0} \in C(\bar{Q})$, such that

$$
y_{a}+\delta \leq G\left(\bar{u}+S^{*} v_{0}\right) \leq y_{b}-\delta,
$$

with a given $\delta>0$. Let the regularization parameter $\varepsilon$ associated with the auxiliary control depend on $\lambda$ as follows:

$$
\varepsilon=c_{0} \lambda^{1+c_{1}}, \quad c_{0}>0, \quad 0 \leq c_{1}<1 .
$$

Again we note that from the Slater condition we deduce the existence of a control $\hat{v} \in L^{2}(Q)$ feasible for all $\left(P B_{\lambda}\right)$ if $\lambda$ is sufficiently small.

Let now $\left\{\lambda_{n}\right\}$ be a sequence of positive real numbers converging to zero as $n \rightarrow \infty$, and let $\left\{v_{n}\right\}$ denote the sequence of associated optimal solutions of $\left(P B_{\lambda_{n}}\right)$. From $\nu>0$ we obtain that

$$
\left\|S^{*} v_{n}\right\|_{L^{2}(\Sigma)}^{2} \leq \frac{2}{\nu} f_{\varepsilon}(\hat{v})
$$

i.e. the boundedness of $\left\{u_{n}\right\}=\left\{S^{*} v_{n}\right\}$ in $L^{2}(\Sigma)$. By a standard argument, we conclude that $\left\{S^{*} v_{n}\right\}$ converges weakly to some $u^{*}$ in $L^{2}(\Sigma)$. The feasibility of $u^{*}$ for $(P B)$ is shown with the help of the assumptions on $\varepsilon(\lambda)$. Since $v_{n}$ is feasible for $\left(P B_{\lambda_{n}}\right)$, i.e.

$$
y_{a} \leq \lambda_{n} v_{n}+G S^{*} v_{n} \leq y_{b},
$$

it is sufficient to show that $\lambda_{n} v_{n}$ converges to zero. With $\varepsilon_{n}>0$ from

$$
\frac{\varepsilon_{n}}{2}\left\|v_{n}\right\|_{L^{2}(Q)}^{2} \leq c
$$

we obtain

$$
\frac{\varepsilon_{n}}{2 \lambda_{n}^{2}}\left\|\lambda_{n} v_{n}\right\|_{L^{2}(Q)}^{2} \leq c
$$

which yields

$$
\left\|\lambda_{n} v_{n}\right\|_{L^{2}(Q)}^{2} \leq \frac{2 c \lambda_{n}^{2}}{\varepsilon_{n}}=\frac{2 c \lambda_{n}^{2}}{c_{0} \lambda_{n}^{1+c_{1}}}=\frac{2 c}{c_{0}} \lambda_{n}^{1-c_{1}}
$$

Hence, $\left\|\lambda_{n} v_{n}\right\|^{2} \rightarrow 0$, for $n \rightarrow \infty$, which implies the feasibility of $u^{*}$ for $(P B)$.

Theorem 3.3. Let $\lambda_{n} \rightarrow 0$ and $\left\{v_{n}\right\}_{n=1}^{\infty}$ be the sequence of optimal controls of $\left(P B_{\lambda_{n}}\right)$. Then, under Assumption 3.2 , the sequence $\left\{S^{*} v_{n}\right\}$ converges strongly in $L^{2}(\Sigma)$ towards the solution $\bar{u}$ of the unregularized problem $(P B)$.

Proof. It is easy to show that the range of $S^{*}: L^{2}(Q) \rightarrow L^{2}(\Sigma)$ is dense in $L^{2}(\Sigma), c f$. the elliptic case discussed in [30]. Further, $C(\bar{Q})$ is dense in $L^{2}(Q)$. We can therefore find a sequence $\left\{v_{k}\right\}$ in $C(\bar{Q})$ such that

$$
\left\|\bar{u}-S^{*} v_{k}\right\|_{L^{2}(\Sigma)} \leq \frac{1}{k} \quad \forall k \in \mathbb{N} .
$$

By Assumption 3.2, we know $G \bar{u} \in C(\bar{Q})$, as well as $G S^{*} v_{k} \in C(\bar{Q})$. Therefore, we can find a positive real number $c$ such that

$$
\left\|G\left(\bar{u}-S^{*} v_{k}\right)\right\|_{C(\bar{Q})} \leq c\left\|\bar{u}-S^{*} v_{k}\right\|_{L^{2}(\Sigma)} \leq \frac{c}{k} \quad \forall k \in \mathbb{N} .
$$

We make again use of an auxiliary sequence $\left\{v_{k}^{0}\right\}$ in $L^{\infty}(Q)$, defined by

$$
v_{k}^{0}=v_{k}+\frac{2 c}{k \delta} v_{0}
$$


where $v_{0}$ denotes the interior Slater point we assumed to exist. The strong convergence of $\left\{S^{*} v_{k}^{0}\right\}$ towards $\bar{u}$ is easily shown:

$$
\left\|S^{*} v_{k}^{0}-\bar{u}\right\|_{L^{2}(\Sigma)} \leq\left\|S^{*} v_{k}-\bar{u}\right\|_{L^{2}(\Sigma)}+\frac{2 c}{k \delta}\left\|S^{*} v_{0}\right\|_{L^{2}(\Sigma)} \rightarrow 0
$$

as $k \rightarrow \infty$. We will prove that for $k$ large enough, there exists $n_{k} \in \mathbb{N}$ such that $v_{k}^{0}$ is feasible for all $\left(P B_{\lambda_{n}}\right)$ with $n \geq n_{k}$. We consider the term $\lambda_{n} v_{k}^{0}+G S^{*} v_{k}^{0}$. Following the steps in [30], we deduce that $v_{k}^{0}$ is feasible for $\left(P B_{\lambda}\right)$, if $\lambda$ is sufficiently small.

The rest of the proof is analogous to the distributed case. By the same steps as in Section 2.2, we obtain $u^{*}=\bar{u}$. Also, from the structure of the objective function, we obtain strong $L^{2}$-convergence $u_{n} \rightarrow \bar{u}$.

\subsection{Numerical experiments}

\subsubsection{An analytic example}

Let us first try the regularization technique on an analytic example similar to the distributed control problem. Again, we consider a one-dimensional problem in $Q=(0,1) \times(0,1)$, referred to as $\left(P B_{\lambda}\right)$. Notice that $\Gamma=$ $\{0\} \cup\{1\}$. With $\alpha=0, \beta=1$ we consider

$$
\min J(y, u):=\frac{1}{2}\left\|y-y_{d}\right\|_{L^{2}(Q)}^{2}+\frac{\nu}{2}\left\|u-u_{d}\right\|_{L^{2}(\Sigma)}^{2}
$$

subject to

$$
\begin{aligned}
y_{t}(x, t)-y_{x x}(x, t) & =f(x, t) & & \text { in } Q \\
y(x, 0) & =0 & & \text { in } \Omega \\
-y_{x}(0, t) & =u(0, t) & & \text { on } \Sigma_{1}=\{0\} \times(0, T) \\
y_{x}(1, t) & =u(1, t) & & \text { on } \Sigma_{2}=\{1\} \times(0, T) \\
& & & \\
y(x, t) & \leq y_{b}(x, t) & & \text { a.e. } \operatorname{in} Q .
\end{aligned}
$$

We define $\hat{y}=\sin (\pi x) \sin (\pi t)$ and specify the data through

$$
\begin{gathered}
y_{d}=\hat{y}+\max (0, \hat{y}-c)-\pi \cos (\pi x)-\pi^{2}(1-t) \sin (\pi x) \\
u_{d}=-\pi \sin (\pi t)+\frac{1}{\nu}(1-t) \\
f=\hat{y}_{t}-\hat{y}_{x x}, \quad y_{b}=\max (c, \hat{y})
\end{gathered}
$$

for a real number $0<c<1$ and $\nu>0$. It is easily verified that

$$
\bar{y}=\hat{y}, \quad \bar{u}=-\pi \sin (\pi t)
$$

is an optimal solution. An adjoint state $p$ and Lagrange multiplier $\mu$, are given by

$$
p=\cos (\pi x)(1-t), \quad \mu=\max (0, \hat{y}-c) .
$$

We point out that uniqueness of $p$ and $\mu$ may not necessarily be expected. Unlike in the distributed case, the gradient equation only holds on $\Sigma$, thus multiple Lagrange multipliers could result in adjoint states differing in the interior and fulfilling the gradient equation. This is supported by the following example in $Q=(0, \pi) \times(0, T)$, $T \in \mathbb{R}^{+}$, with $\bar{y}=y_{d}=y_{b}$. Here, adjoint state and Lagrange multiplier have to satisfy

$$
\begin{aligned}
-p_{t}-\Delta p & =\mu_{b}, \\
p(x, T) & =0, \quad \mu_{b} \geq 0 . \\
\partial_{n} p & =0,
\end{aligned}
$$

It is easily verified that this is true for all $p$ obtained by

$$
p=\kappa \sin ^{2}(x)(T-t)+c(T-t),
$$


with $c$ fixed and corresponding $\mu_{b}$ for all $0<\kappa<\frac{c}{2 T}$. The constant $c>0$ is introduced to guarantee $\mu_{b}=-p_{t}-\Delta p \geq 0$.

For our numerical experiments, we have again chosen an example with essentially bounded optimal control and continuous state function. We use a solution algorithm very similar to Algorithm 1 applied to the distributed problem. In analogy to Section 2.3 , we obtain

$$
\begin{aligned}
& \mu_{a}=\max \left(0, \frac{1}{\lambda} q+\frac{\varepsilon}{\lambda^{2}}\left(y_{a}-\bar{y}\right)\right) \\
& \mu_{b}=\max \left(0,-\frac{1}{\lambda} q+\frac{\varepsilon}{\lambda^{2}}\left(\bar{y}-y_{b}\right)\right) .
\end{aligned}
$$

We define the active sets $\mathcal{A}_{a}$ and $\mathcal{A}_{b}$,

$$
\begin{aligned}
& \mathcal{A}_{a}(y, q):=\left\{(x, t) \in Q \mid q+\frac{\varepsilon}{\lambda}\left(y_{a}-y\right)>0 \text { a.e. in } Q\right\} \\
& \mathcal{A}_{b}(y, q):=\left\{(x, t) \in Q \mid-q+\frac{\varepsilon}{\lambda}\left(y-y_{b}\right)>0 \text { a.e. in } Q\right\}
\end{aligned}
$$

then Algorithm 2 is given by

\section{Algorithm 2.}

(1) Initialize $\left(y_{0}, u_{0}, v_{0}, p_{0}, q_{0}, \mu_{a, 0}, \mu_{b, 0}\right)$ and set $n=0$.

(2) Determine the sets $\mathcal{A}_{a, n}=\mathcal{A}_{a}\left(y_{n}, q_{n}\right)$ and $\mathcal{A}_{b, n}=\mathcal{A}_{b}\left(y_{n}, q_{n}\right)$.

(3) Find $\left(y_{n+1}, u_{n+1}, v_{n+1}, p_{n+1}, q_{n+1}, \mu_{a_{n+1}}, \mu_{b_{n+1}}\right)$ that solve

$$
(O P T)\left\{\begin{aligned}
y_{n+1} & =S u_{n+1} \\
u_{n+1} & =S^{*} v_{n+1} \\
\beta p_{n+1} & =S^{*}\left(y_{n+1}-y_{d}+\mu_{b_{n+1}}-\mu_{a_{n+1}}\right) \\
q_{n+1} & =S\left(\beta p_{n+1}+\nu u_{n+1}\right) \\
0 & =\varepsilon v_{n+1}+q_{n+1}+\lambda\left(\mu_{b_{n+1}}-\mu_{a_{n+1}}\right) \\
\mu_{a_{n+1}} & =\left(\frac{1}{\lambda} q_{n+1}+\frac{\varepsilon}{\lambda^{2}}\left(y_{a}-y_{n+1}\right)\right)_{\mathcal{X}_{\mathcal{A}_{a, n}}} \\
\mu_{b_{n+1}} & =\left(-\frac{1}{\lambda} q_{n+1}+\frac{\varepsilon}{\lambda^{2}}\left(y_{n+1}-y_{b}\right)\right)_{\mathcal{X}_{\mathcal{A}_{b, n}}} .
\end{aligned}\right.
$$

(4) If $n \geq 1$ and $\mathcal{A}_{a, n+1}=\mathcal{A}_{a, n}$ and $\mathcal{A}_{b, n+1}=\mathcal{A}_{b, n}$ STOP, else set $n=n+1$ and go to step (2).

As before, the initial values for the algorithm are determined as the solution of a problem without state constraints, using $\mathcal{A}_{a,-1}=\mathcal{A}_{b,-1}=\emptyset$. Note that Algorithm 2 can be adjusted to the case of a general shift function $u_{d} \neq 0 \in L^{2}(\Sigma)$ by setting

$$
\begin{aligned}
& u_{n+1}=S^{*} v_{n+1}+u_{d} \\
& q_{n+1}=S\left(\beta p_{n+1}+\nu\left(u_{n+1}-u_{d}\right)\right)
\end{aligned}
$$

in each iteration, if $u_{d}$ is not eliminated beforehand. We proceed similarly to the distributed case. We use again a standard discretization of the Laplace operator and an implicit Euler scheme for solving all appearing parabolic equations. We try different values of $\lambda$ and summarize the results for $c=0.5$ and $\nu=10^{-4}$ in Table 2. Figure 8 shows the convergence behavior of the control depending on the Lavrentiev parameter. From the results, we deduce that a Lavrentiev parameter of approximately $10^{-7}$ or $10^{-8}$ seems to be appropriate. Figures 6 and 7 show the numerical results for $\lambda=10^{-8}$. Graphically, there is no difference between the regularized solution and the true solution. The Tikhonov parameter $\varepsilon$ was chosen $\varepsilon=\lambda^{1.5}$, and thus clearly satisfies the convergence criterion (3.11). 


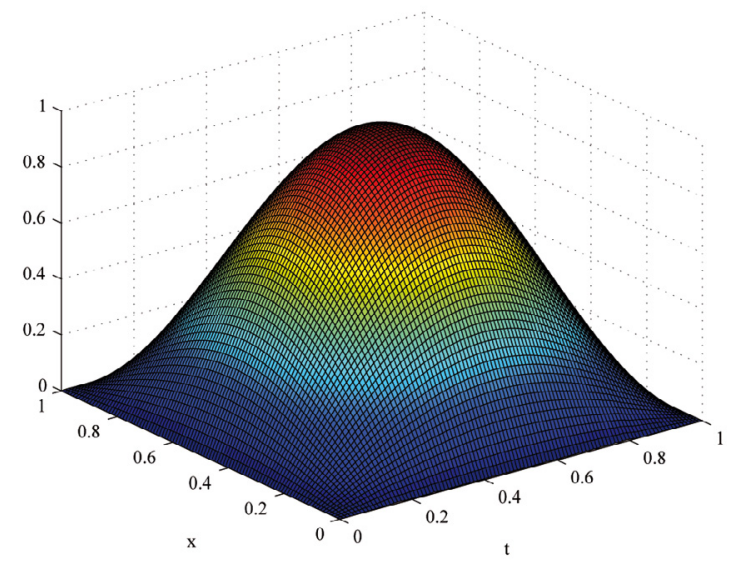

FiguRE $6 . \bar{y}_{\tau h}$ at $c=0.5$.

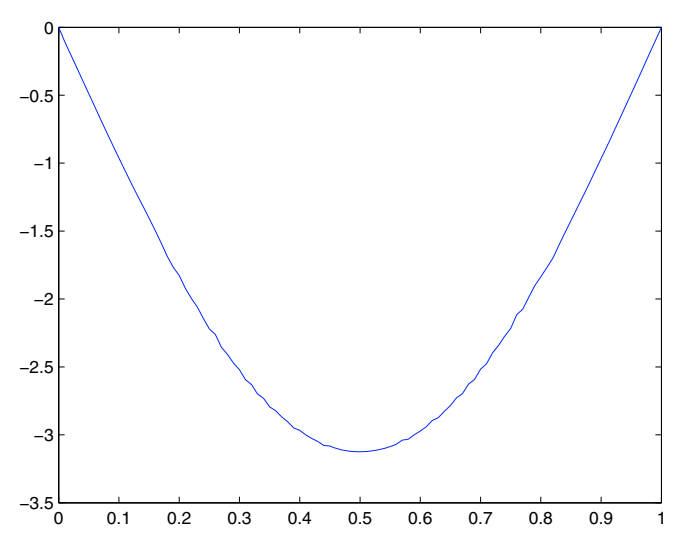

Figure $7 . \bar{u}_{\tau h}$ at $c=0.5$.

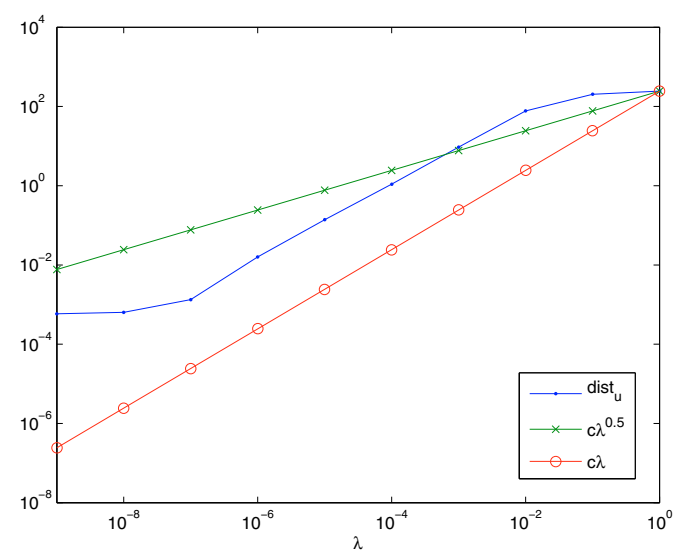

FiguRE 8. Relative $L^{2}$-error of $\bar{u}_{\tau h}$.

TABle 2. Convergence behavior of Algorithm 2 for $\lambda \rightarrow 0$.

\begin{tabular}{|c|c|c|c|}
\hline$\lambda$ & iter & dist $_{y}$ & dist $_{u}$ \\
\hline $10^{+0}$ & 5 & $3.0964 \mathrm{e}+01$ & $2.4322 \mathrm{e}+02$ \\
\hline $10^{-1}$ & 5 & $2.9285 \mathrm{e}+01$ & $2.0309 \mathrm{e}+02$ \\
\hline $10^{-2}$ & 6 & $1.0430 \mathrm{e}+01$ & $7.6978 \mathrm{e}+01$ \\
\hline $10^{-3}$ & 7 & $9.8874 \mathrm{e}-01$ & $9.4543 \mathrm{e}+00$ \\
\hline $10^{-4}$ & 8 & $9.0601 \mathrm{e}-02$ & $1.0993 \mathrm{e}+00$ \\
\hline $10^{-5}$ & 9 & $7.0612 \mathrm{e}-03$ & $1.3800 \mathrm{e}-01$ \\
\hline $10^{-6}$ & 10 & $7.2127 \mathrm{e}-04$ & $1.5986 \mathrm{e}-02$ \\
\hline $10^{-7}$ & 8 & $7.8225 \mathrm{e}-05$ & $1.3336 \mathrm{e}-03$ \\
\hline $10^{-8}$ & 9 & $2.4731 \mathrm{e}-05$ & $6.3628 \mathrm{e}-04$ \\
\hline $10^{-9}$ & 9 & $2.3627 \mathrm{e}-05$ & $5.8503 \mathrm{e}-04$ \\
\hline
\end{tabular}




\subsubsection{The Betts and Campbell heat transfer problem}

As pointed out in the introduction, the benchmark problem by Betts and Campbell in [7] inspired our research. However, since this problem is formulated with Dirichlet boundary conditions it does not fit into the context of this paper without adaption. Since the analysis of Dirichlet boundary conditions would go much beyond the scope of this paper, $c f$. [2], we approximate the Dirichlet conditions by Robin type boundary conditions and therefore consider a different, but similar problem. We will refer to this problem as approximation to the Betts and Campbell heat transfer problem. We do point out that we choose the boundary conditions $\partial_{n} y+\alpha y=\beta u$ with fixed values of $\alpha=\beta=10^{6}$, since this seems large enough from a naive, heuristic point of view. Hence, both values do not serve as additional method-related regularization parameters, but become problem-given constants instead. We do not aim at a convergence analysis for $\alpha, \beta \rightarrow \infty$. However, we point out that the approximation of Dirichlet boundary conditions by Robin boundary conditions is not unusual in practice, and we mention the work in [2] by Arada et al. where such an approximation is considered in a state-constrained context. Hence, we choose the data in $\left(P B_{1 D}\right)$ as follows:

$$
\begin{gathered}
\Omega=(0, \pi), \quad Q=\Omega \times(0,5), \quad \nu=10^{-3}, \\
y_{d}=0, \quad u_{d}=0, \quad y_{a}=\sin (x) \sin \left(\frac{\pi t}{5}\right)-0.7 .
\end{gathered}
$$

Note that $y=y_{a}$ is only possible where the bound $y_{a}$ satisfies the differential equation, i.e. $y_{a, t}-\Delta y_{a}=0$. Inserting the expression for $y_{a}$ yields

$$
0=\frac{\pi}{5} \sin (x) \cos \left(\frac{\pi}{5} t\right)+\sin (x) \sin \left(\frac{\pi}{5} t\right)=\sin (x)\left(\frac{\pi}{5} \cos \left(\frac{\pi}{5} t\right)+\sin \left(\frac{\pi}{5} t\right)\right),
$$

which on the given domain is only true on the sets of measure zero,

$$
\left\{(x, t) \mid x=\frac{\pi}{2}\right\} \text { and }\left\{(x, t) \mid t=\frac{5}{\pi}\left(\pi-\cot \left(\frac{5}{\pi}\right)\right)\right\} \text {. }
$$

This implies that the Lagrange multiplier associated with the constraint is a Borel measure.

As mentioned above, we set $\alpha=\beta=10^{6}$ to approximate the Dirichlet boundary conditions $u(0, t)=u_{0}(t)$, $u(\pi, t)=u_{\pi}(t)$.

Unfortunately, we do not know the analytic solution to the problem. To obtain a reference solution we solve this problem - with Robin type boundary conditions - with the Matlab code quadprog. The solution is shown in Figures 9 and 10 and is referred to as $u_{\text {unreg, }}, y_{\text {unreg, }}$, respectively. We point out that we do not know a priori if the optimal control is regular enough to guarantee a continuous optimal state. Yet, the numerical results obtained by quadprog indicate that $\bar{u}(t)=\left\{u_{0}(t), u_{\pi}(t)\right\}$ belongs to $L^{\infty}(0,5) \times L^{\infty}(0,5)$. Hence, the convergence analysis derived previously should be applicable.

Figures 11 and 12 show the results of the regularization method on a grid with $n_{x}=101, n_{t}=101$, with regularization parameters $\lambda=10^{-8}$ and $\varepsilon=10^{-12}$. In Figures 13 and 14, we show in addition the Lagrange multipliers for the regularized as well as the unregularized problem. A direct comparison of both multipliers is difficult, since they are obtained by completely different methods and are also different in nature. While in the unregularized solution the constraints are only active at $x=\frac{\pi}{2}$ for a certain interval in time, the regularization yields a multiplier which is nonzero on a subset of $Q$ with positive measure. Moreover, the unregularized multiplier admits peaks towards the boundary of the active time interval which are characteristic for a measure and disappear in the regularized case. To still give a comparison, we scale both multipliers such that their regular parts have comparable size. More precisely, both multipliers are scaled such that their value at $(x, t)=\left(\frac{\pi}{2}, \frac{5}{2}\right)$, which seems to be roughly the center of the active set, is equal to one. Their values at $x=\frac{\pi}{2}$ in time are shown in Figure 15.

We compare the results for different values of $\lambda$, again choosing $\varepsilon=\lambda^{1.5}$, to the results obtained by quadprog for the unregularized control problem. Table 3 displays the relative $L^{2}$-difference, and Figure 17 illustrates 


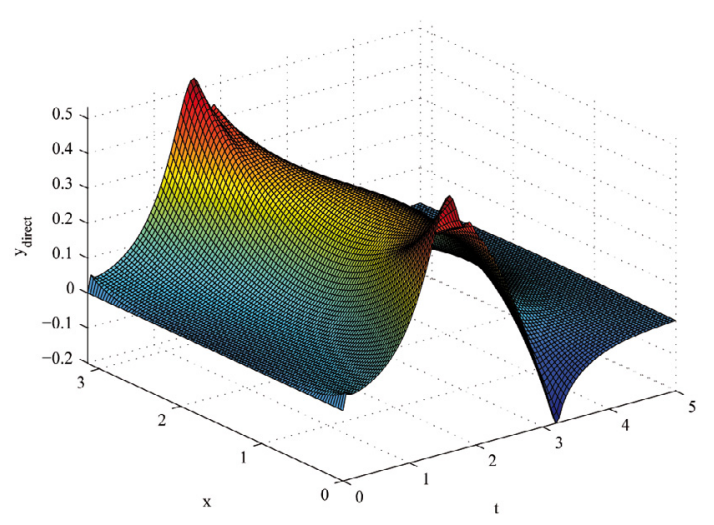

FiguRE 9. $\bar{y}_{\text {unreg }}$.

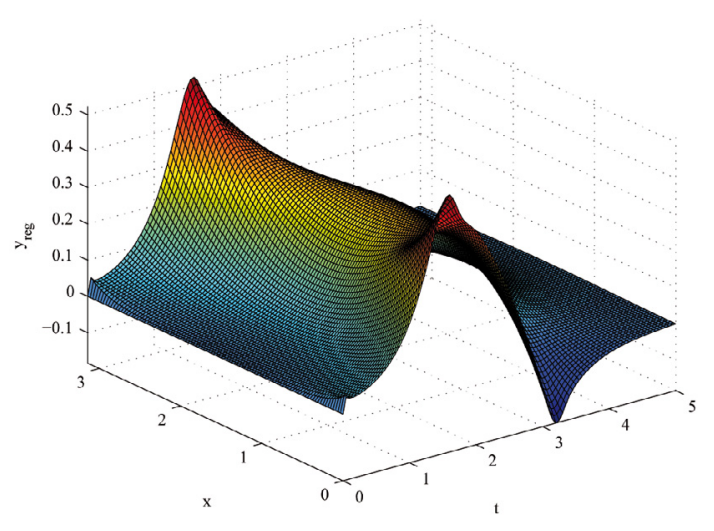

FIGURE 11. $\bar{y}_{\text {reg. }}$.

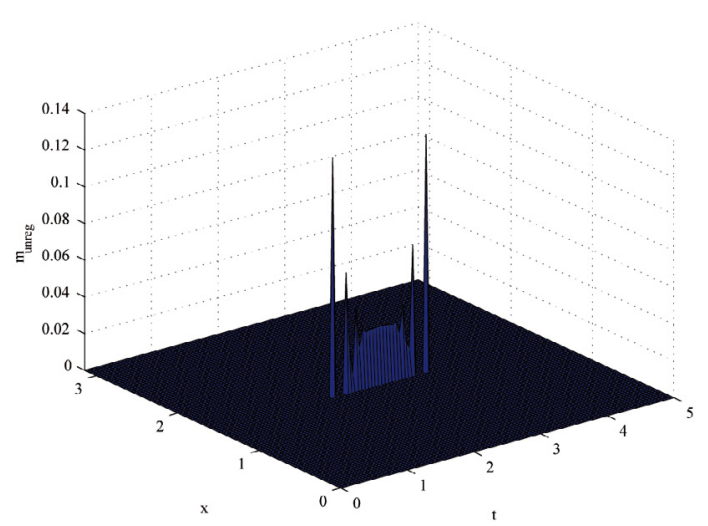

FIGURE 13. Unregularized multiplier $\mu_{\text {unreg }}$.

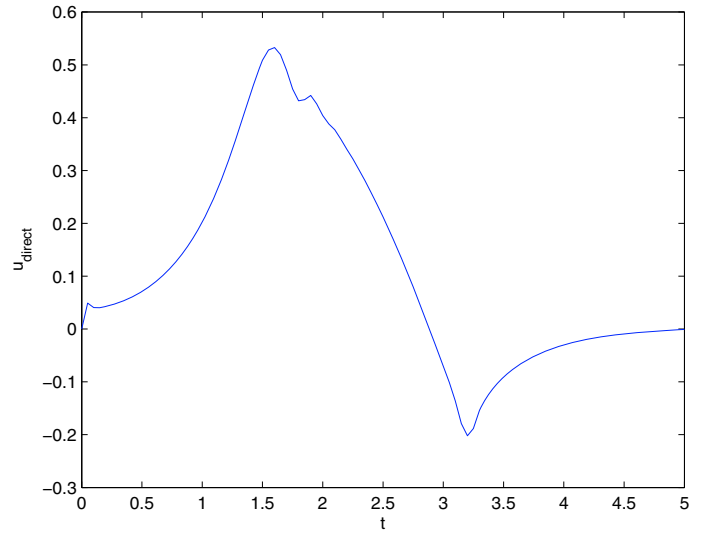

FiguRE 10. $\bar{u}_{\text {unreg }}$.

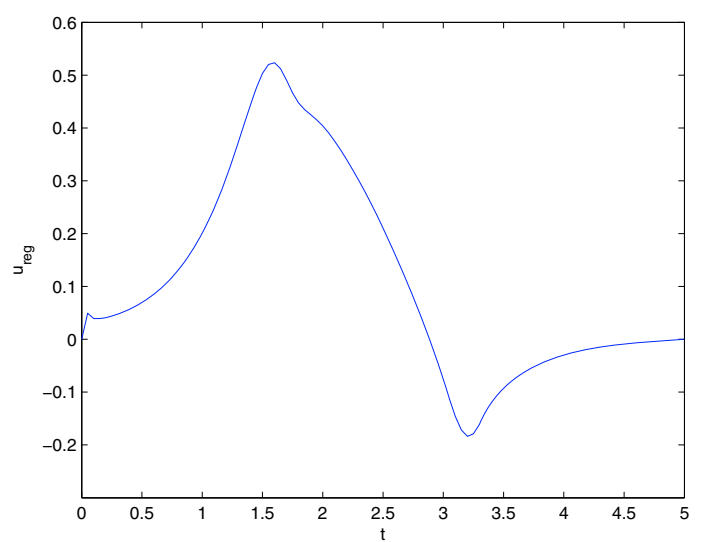

FiguRE $12 . \bar{u}_{\text {reg }}$.

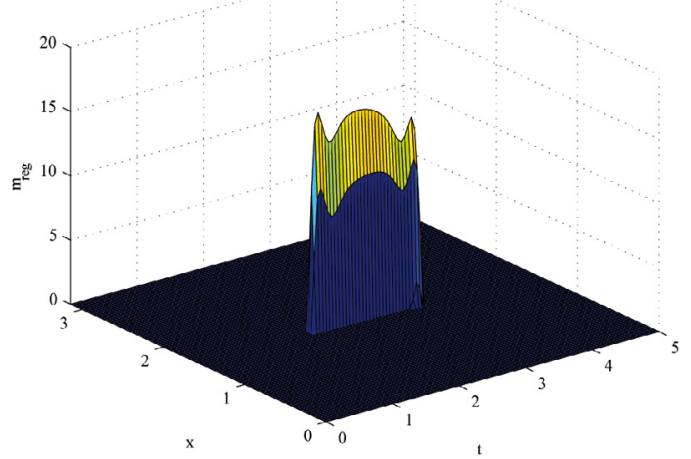

FigURE 14. Regularized multiplier $\mu_{\text {reg }}$. 


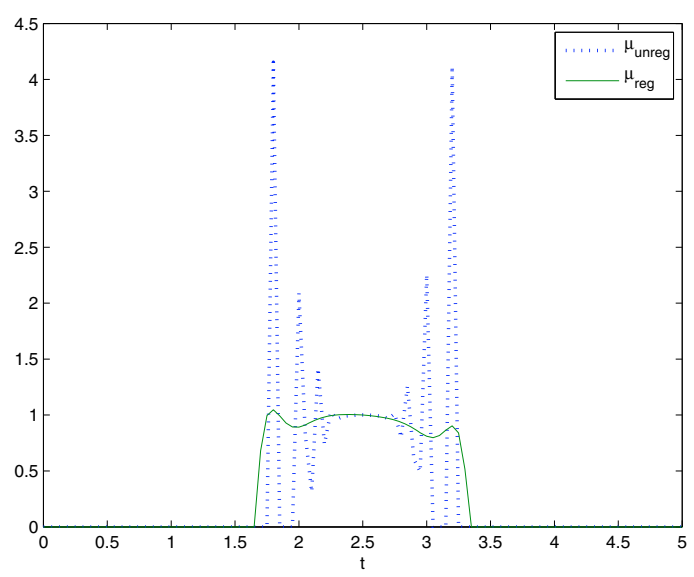

Figure 15. Comparison of regularized and unregularized multipliers at $x=\frac{\pi}{2}$.

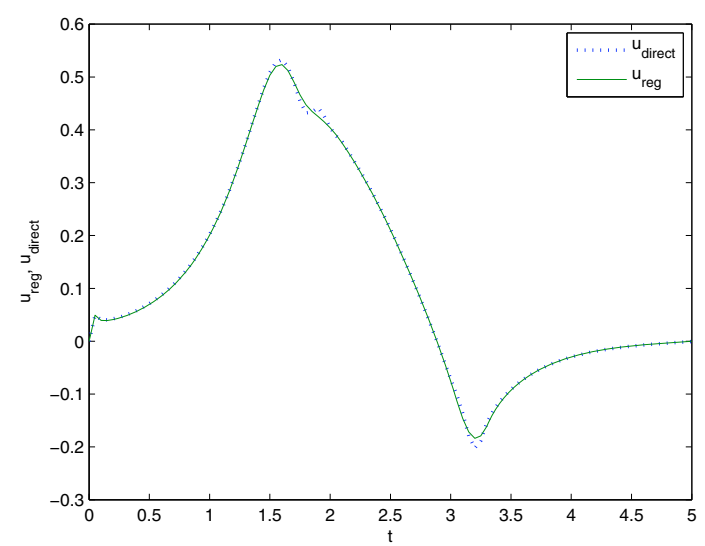

FiguRE 16. $\bar{u}_{\text {unreg }}$ vs. $\bar{u}_{\text {reg. }}$.

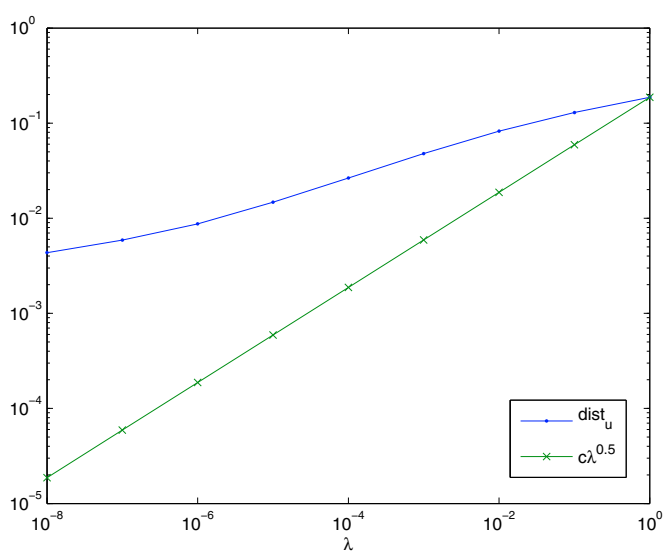

FIGURE 17. Relative $L^{2}$-error of $\bar{u}_{\text {reg }}$.

convergence graphically. Referring to the optimal control pertaining to $\lambda=10^{-8}$ as $u_{\text {reg }}$, Figure 16 shows the controls $u_{\text {unreg }}$ and $u_{\text {reg }}$ in comparison. The explicit choice of the dependence of $\lambda$ and $\varepsilon$ is experimental, but based on the results of the last example and the analytic relationship between $\lambda$ and $\varepsilon$.

Just like for the analytic example, we observe that the approximation improves as the regularization parameters decrease, but for this particular problem there is still a significant difference between the two solutions. To rule out any bias imposed by the different solution methods, quadprog on the one hand and Algorithm 3 on the other hand, we solve the regularized problem

$$
\begin{aligned}
\min J(y, w, v)=\frac{1}{2}\|y\|_{L^{2}(Q)}^{2}+\frac{\nu}{2}\|\beta w\|_{L^{2}(\Sigma)}^{2}+\frac{\epsilon}{2}\|v\|_{L^{2}(Q)}^{2}, & \\
y_{t}-\Delta y= & 0, \quad-w_{t}-\Delta w=v \\
y(\cdot, 0)= & 0, \quad w(\cdot, T)=0 \\
\partial_{n} y+\alpha y= & \beta^{2} w, \quad \partial_{n} w+\alpha w=0 \\
& \lambda v+y \geq y_{a}
\end{aligned}
$$


TABLE 3. Direct vs. regularized indirect solution.

\begin{tabular}{|c|c|c|c|}
\hline$\lambda$ & iter & dist $_{y}$ & dist $_{u}$ \\
\hline $1.00 \mathrm{e}+00$ & 4 & $3.2479 \mathrm{e}-02$ & $1.8711 \mathrm{e}-01$ \\
\hline $1.00 \mathrm{e}-01$ & 4 & $2.2563 \mathrm{e}-02$ & $1.2968 \mathrm{e}-01$ \\
\hline $1.00 \mathrm{e}-02$ & 5 & $1.4152 \mathrm{e}-02$ & $8.2507 \mathrm{e}-02$ \\
\hline $1.00 \mathrm{e}-03$ & 5 & $8.0149 \mathrm{e}-03$ & $4.7934 \mathrm{e}-02$ \\
\hline $1.00 \mathrm{e}-04$ & 6 & $4.2467 \mathrm{e}-03$ & $2.6492 \mathrm{e}-02$ \\
\hline $1.00 \mathrm{e}-05$ & 7 & $2.1838 \mathrm{e}-03$ & $1.4752 \mathrm{e}-02$ \\
\hline $1.00 \mathrm{e}-06$ & 9 & $1.1299 \mathrm{e}-03$ & $8.7261 \mathrm{e}-03$ \\
\hline $1.00 \mathrm{e}-07$ & 9 & $6.2461 \mathrm{e}-04$ & $5.8978 \mathrm{e}-03$ \\
\hline $1.00 \mathrm{e}-08$ & 11 & $3.8864 \mathrm{e}-04$ & $4.3430 \mathrm{e}-03$ \\
\hline
\end{tabular}

with quadprog. This means we apply a direct solution method, or Discretize then Optimize method, since the problem is discretized and the resulting NLP is then solved. On the other hand, deriving optimality conditions and solving the discretized optimality system is also known as Optimize then Discretize, or indirect solution approach. The active set strategy from Algorithms 1-3 is such an indirect method. We point out that we do not intend to compare the numerical performance of the two solution strategies, but use the direct solution as a reference solution where an analytic expression is not at hand. We experimented with different choices of $\varepsilon$ for a fixed $\lambda=10^{-8}$. Figures 18 and 19 show the comparison of the unregularized solution $\bar{u}$ obtained with quadprog, and the regularized solution $\bar{u}_{\text {reg }}$ obtained with quadprog for $\varepsilon=500 \lambda^{1.5}$ and $\varepsilon=\lambda^{1.5}$, respectively, on a grid with $n_{x}=n_{t}=51$. Figure 18 shows that the regularization technique can yield results very similar to the unregularized solution, and also indicates that the choice of $\varepsilon$ influences the quality of the approximation quite strongly. For the indirect method, parameter tuning for $\varepsilon$ proves to be difficult. Therefore, other methods to solve the optimality system, for example adaptive grid techniques, should be considered.

Remark 3.4. So far, all experiments in this section have been done for Robin boundary conditions with $\alpha=\beta=10^{6}$, as pointed out initially. Our results show the typical solution behavior of the original Betts and Campbell heat transfer problem. In order to relate our results to the original Dirichlet-type problem more precisely, we have also applied the regularization technique with parameters $\lambda=10^{-8}$ and $\varepsilon=10^{-12}$ to the original problem, and solved the resulting optimal control problem with the Discretize then Optimize approach utilizing quadprog. On a grid with $n_{x}=n_{t}=51$, we obtain a maximum difference of order $10^{-3}$ in a direct comparison of the values of the control at the grid points. This indicates, without analysis, that the Robin parameters have been chosen well enough to preserve the typical problem behavior, since $10^{-3}$ is significantly smaller than the best precision that can be expected on a time grid of diameter $\frac{5}{50}=0.1$.

Further experiments have eventually led to a modified regularization technique, still subject to further research. We recall that we have substituted $u=S^{*} v=\left.\beta z\right|_{\Sigma}$, which leads to a term $\frac{\nu}{2}\|u\|_{L^{2}(\Sigma)}^{2}=\frac{\nu}{2}\|\beta z\|_{L^{2}(\Sigma)}^{2}$ in the objective function of the regularized problem. Since $z$ is defined on $Q$, it seems reasonable to replace the term $\frac{\nu}{2}\|u\|_{L^{2}(\Sigma)}^{2}$ by $\frac{\nu}{2}\|\beta z\|_{L^{2}(Q)}^{2}$ instead. This changes equation (3.8) in the optimality system to

$$
\begin{aligned}
q_{t}-\Delta q & =\beta^{2} \nu z \\
q(\cdot, 0) & =0 \\
\partial_{n} q+\alpha q & =\beta^{2} p,
\end{aligned}
$$

which sometimes yields better results than the original regularization technique.

\subsection{Application of a method by Ito and Kunisch}

Without further analysis, we have also applied the regularization method considered in [15] for elliptic distributed control problems, based on a penalization of the pointwise state constraints, to our model problem 


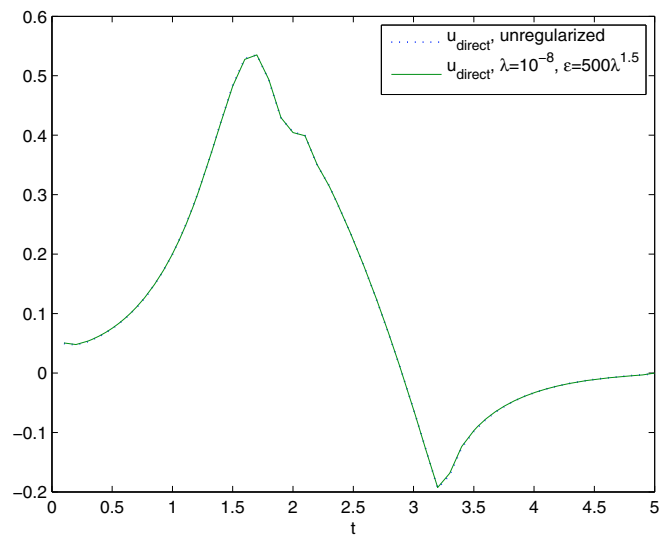

FIGURE 18. $\bar{u}_{\text {unreg, direct }}$ vs. $\bar{u}_{\text {reg, direct }}$.

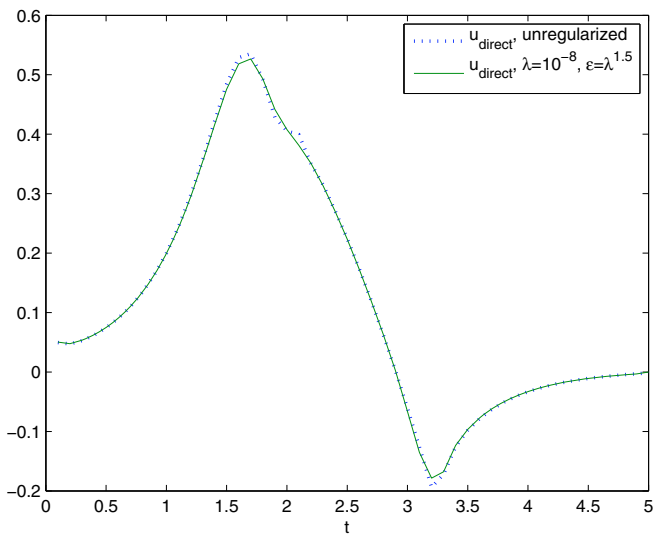

FIGURE 19. $\bar{u}_{\text {unreg, direct }}$ vs. $\bar{u}_{\text {reg, direct }}$.

with boundary control, $(P B)$. Following [15], we note that technically (i.e. tacitly assuming $\mu_{a}$ and $\mu_{b}$ to be functions) one finds for the Lagrange multipliers

$$
\mu_{a}=\max \left(0, \mu_{a}-\mu_{b}+y_{a}-y\right), \quad \mu_{b}=\max \left(0, \mu_{b}-\mu_{a}+y-y_{b}\right),
$$

which, for regularization, is replaced in [15] by

$$
\mu_{a}=\max \left(0, \bar{\mu}_{a}-\bar{\mu}_{b}+\gamma\left(y_{a}-y\right)\right), \quad \mu_{b}=\max \left(0, \bar{\mu}_{b}-\bar{\mu}_{a}+\gamma\left(y-y_{b}\right)\right),
$$

with $\gamma>0$ and arbitrary $\bar{\mu}_{a}, \bar{\mu}_{b} \in L^{2}(Q)$. With the definition of the active sets

$$
\begin{aligned}
& \mathcal{A}_{a}(y):=\left\{(x, t) \in Q \mid \bar{\mu}_{a}-\bar{\mu}_{b}+\gamma\left(y_{a}-y\right)>0 \text { a.e. in } Q\right\} \\
& \mathcal{A}_{b}(y):=\left\{(x, t) \in Q \mid \bar{\mu}_{b}-\bar{\mu}_{a}+\gamma\left(y-y_{b}\right)>0 \text { a.e. in } Q\right\},
\end{aligned}
$$

the solution algorithm from [15] applied to our model problem now reads:

\section{Algorithm 3.}

(1) Choose $\left(y_{0}, u_{0}, p_{0}, \mu_{a, 0}, \mu_{b, 0}\right)$ and set $n=0$.

(2) Determine the sets $\mathcal{A}_{a, n}=\mathcal{A}_{a}\left(y_{n}, \mu_{a, n}\right)$ and $\mathcal{A}_{b, n}=\mathcal{A}_{b}\left(y_{n}, \mu_{b, n}\right)$ by (3.12) and (3.13).

(3) Find $\left(y_{n+1}, u_{n+1}, p_{n+1}, \mu_{a_{n+1}}, \mu_{b_{n+1}}\right)$ that solve

$$
(O P T)\left\{\begin{aligned}
y_{n+1} & =S u_{n+1} \\
\beta p_{n+1} & =S^{*}\left(y_{n+1}-y_{d}+\mu_{b_{n+1}}-\mu_{a_{n+1}}\right) \\
0 & =\nu\left(u_{n+1}-u_{d}\right)+\beta p_{n+1} \\
\mu_{a_{n+1}} & =\bar{\mu}_{a}-\bar{\mu}_{b}+\gamma\left(y_{a}-y_{n+1}\right) \mathcal{X}_{\mathcal{A}_{a, n}} \\
\mu_{b_{n+1}} & =\bar{\mu}_{b}-\bar{\mu}_{a}+\gamma\left(y_{n+1}-y_{b}\right) \mathcal{X}_{\mathcal{A}_{b, n}} .
\end{aligned}\right.
$$

(4) If $n \geq 1$ and $\mathcal{A}_{a, n+1}=\mathcal{A}_{a, n}$ and $\mathcal{A}_{b, n+1}=\mathcal{A}_{b, n}$ STOP, else set $n=n+1$ and go to step (2).

Again, the algorithm is initialized with the solution pertaining to a problem without inequality constraints on the state. Choosing $\bar{\mu}_{a}=\bar{\mu}_{b}=0$, one step of the algorithm corresponds to solving the optimality system for the problem

$$
\min J(y, u)+\frac{1}{2 \gamma}\left(\iint_{\mathcal{A}_{a, n}}\left|\gamma\left(y_{a}-y\right)\right|^{2} \mathrm{~d} x \mathrm{~d} t+\iint_{\mathcal{A}_{b, n}}\left|\gamma\left(y-y_{b}\right)\right|^{2} \mathrm{~d} x \mathrm{~d} t\right)
$$


TABLE 4. Convergence behavior of the penalization method for $\gamma \rightarrow \infty$.

\begin{tabular}{|c|c|c|c|}
\hline$\gamma$ & iter & dist $_{y}$ & dist $_{u}$ \\
\hline $1.00 \mathrm{e}+00$ & 4 & $1.4568 \mathrm{e}-03$ & $6.9998 \mathrm{e}-03$ \\
\hline $1.00 \mathrm{e}+01$ & 5 & $3.4691 \mathrm{e}-04$ & $1.6893 \mathrm{e}-03$ \\
\hline $1.00 \mathrm{e}+02$ & 6 & $5.7434 \mathrm{e}-05$ & $6.0104 \mathrm{e}-04$ \\
\hline $1.00 \mathrm{e}+03$ & 7 & $2.7446 \mathrm{e}-05$ & $6.0727 \mathrm{e}-04$ \\
\hline $1.00 \mathrm{e}+04$ & 8 & $2.3593 \mathrm{e}-05$ & $5.8265 \mathrm{e}-04$ \\
\hline $1.00 \mathrm{e}+05$ & 9 & $2.3760 \mathrm{e}-05$ & $5.7997 \mathrm{e}-04$ \\
\hline
\end{tabular}

subject to

$$
\begin{aligned}
y_{t}-\Delta y & =0 \\
y(\cdot, 0) & =0 \\
\partial_{\nu} y+\alpha y & =\beta u .
\end{aligned}
$$

We now test Algorithm 3 on our analytic model problem for different values of $\gamma$. We expect convergence towards the unregularized solution for $\gamma \rightarrow \infty$. The results are summarized in Table 4 . We point out that there is little difference in the accuracy of the solution that can be obtained by the Lavrentiev-based regularization method and the method based on penalization. Considering the numerical performance, a drawback of Lavrentiev regularization for boundary control is the fact that the number of PDEs that need to be solved in each iteration doubles compared to the unregularized problem. This is avoided in the method in [15]. The meaning of the two regularization parameters $\gamma$ and $\lambda$ cannot easily be compared, hence, we study the number of iterations needed with respect to the accuracy obtained in the solution. It appears that a comparable level of accuracy is reached by about the same number of iterations. For example, the Lavrentiev regularization method needs 9 iterations to obtain a relative error of $5.8503 \mathrm{e}-04$ in the control $u$. After the same number of iterations, the penalization method results in an error of $5.7997 \mathrm{e}-04$. Taking into account the number of PDEs to be solved in each iteration, the numerical cost of the penalization method is less for this particular problem. In [15], a continuation method based on penalization depending on $\gamma$ has been introduced that effectively reduces the number of iterations. However, applied to our reference problem, we did not see a significant change. We also applied the penalization method to the Betts and Campbell heat transfer problem and observe the same type of behavior. More precisely, applying Lavrentiev regularization, after 11 iterations the relative $L^{2}$-distance to the Discretize Then Optimize solution was $4.3430 \mathrm{e}-03$ for the control $u$ and 3.8864 e-04 for $y$, as opposed to $4.3489 \mathrm{e}-03$ for $u$ and $3.9098 \mathrm{e}-04$ for $y$ using penalization. Graphically, we could not see any difference between the two solutions.

In conclusion, the numerical tests in this section suggest that the Lavrentiev regularization method yields satisfying results, comparable in accuracy to those of the penalization method by Ito and Kunisch. Numerically, in the case of boundary control, the method of Ito and Kunisch is faster than our Lavrentiev-type technique, that is more analysis driven. Our technique is useful to preserve the structure of a state-constrained problem that is, in addition, equivalent to a control-constrained one with regular Lagrange multipliers. The drawback is, that the number of PDEs is increased, hence the implementation is more difficult and the numerical cost is higher.

Acknowledgements. The authors wish to thank John Betts for sharing his expertise and for introducing the first author to direct transcription methods during her internship at the Boeing Company. Particularly, we are grateful for the discussions and support on the benchmark problem in [7], that originally motivated our interest in the regularization of state-constrained boundary control problems. 


\section{REFERENCES}

[1] N. Arada and J.P. Raymond, Optimal control problems with mixed control-state constraints. SIAM J. Control 39 (2000) 1391-1407.

[2] N. Arada, H. El Fekih and J.-P. Raymond, Asymptotic analysis of some control problems. Asymptotic Anal. 24 (2000) $343-366$.

[3] M. Bergounioux and K. Kunisch, Primal-dual active set strategy for state-constrained optimal control problems. Comput. Optim. Appl. 22 (2002) 193-224.

[4] M. Bergounioux, K. Ito and K. Kunisch, Primal-dual strategy for constrained optimal control problems. SIAM J. Control Opt. 37 (1999) 1176-1194.

[5] M. Bergounioux, M. Haddou, M. Hintermüller and K. Kunisch, A comparison of a Moreau-Yosida-based active set strategy and interior point methods for constrained optimal control problems. SIAM J. Optim. 11 (2000) 495-521.

[6] J.T. Betts and S.L. Campbell, Discretize Then Optimize. Technical Report M\&CT-TECH-03-01, Phantom Works, Mathematics \& Computing Technology. A Division of The Boeing Company (2003).

[7] J.T. Betts and S.L. Campbell, Discretize then Optimize, in Mathematics in Industry: Challenges and Frontiers A Process View: Practice and Theory, D.R. Ferguson and T.J. Peters Eds., SIAM Publications, Philadelphia (2005).

[8] J.T. Betts, S.L. Campbell and A. Englesone, Direct transcription solution of optimal control problems with higher order state constraints: theory vs. practice. Optim. Engineering 8 (2007) 1-19.

[9] E. Casas, Boundary control of semilinear elliptic equations with pointwise state constraints. SIAM J. Control Opt. 31 (1993) 993-1006.

[10] E. Casas, Pontryagin's principle for state-constrained boundary control problems of semilinear parabolic equations. SIAM J. Control Opt. 35 (1997) 1297-1327.

[11] K. Deckelnick and M. Hinze, Convergence of a finite element approximation to a state constrained elliptic control problem. SIAM J. Numer. Anal. 45 (2007) 1937-1953.

[12] M. Hintermüller, K. Ito and K. Kunisch, The primal-dual active set strategy as a semismooth Newton method. SIAM J. Optim. 13 (2003) 865-888.

[13] M. Hintermüller, F. Tröltzsch and I. Yousept, Mesh-independence of semismooth Newton methods for Lavrentiev-regularized state constrained nonlinear optimal control problems. Numer. Math. 108 (2008) 571-603.

[14] K. Ito and K. Kunisch, Augmented Lagrangian methods for nonsmooth, convex optimization in Hilbert spaces. Nonlinear Anal. Theory Methods Appl. 41 (2000) 591-616.

[15] K. Ito and K. Kunisch, Semi-smooth Newton methods for state-constrained optimal control problems. Systems Control Lett. 50 (2003) 221-228.

[16] S. Kameswaran and L.T. Biegler, Advantages of Nonlinear Programming Based Methodologies for Inequality Path Constrained Optimal Control Problems - An Analysis of the Betts and Campbell Heat Conduction Problem. Technical report, Chemical Engineering Department Carnegie Mellon, University Pittsburgh, USA (2005).

[17] K. Kunisch and A. Rösch, Primal-dual active set strategy for a general class of constrained optimal control problems. SIAM J. Optim. 13 (2002) 321-334.

[18] J.L. Lions, Optimal Control of Systems Governed by Partial Differential Equations. Springer-Verlag, Berlin (1971).

[19] C. Meyer and F. Tröltzsch, On an elliptic optimal control problem with pointwise mixed control-state constraints, in Recent Advances in Optimization, Proceedings of the 12th French-German-Spanish Conference on Optimization, Avignon, September 20-24, 2004, A. Seeger Ed., Lectures Notes in Economics and Mathematical Systems, Springer-Verlag (2005).

[20] C. Meyer, A. Rösch and F. Tröltzsch, Optimal control of PDEs with regularized pointwise state constraints. Comput. Optim. Appl. 33 (2006) 209-228.

[21] C. Meyer, U. Prüfert and F. Tröltzsch, On two numerical methods for state-constrained elliptic control problems. Optim. Methods Software 22 (2007) 871-899.

[22] I. Neitzel and F. Tröltzsch, On convergence of regularization methods for nonlinear parabolic optimal control problems with control and state constraints. Technical Report 24-03, SPP 1253 (2008).

[23] U. Prüfert, F. Tröltzsch and M. Weiser, The convergence of an interior point method for an elliptic control problem with mixed control-state constraints. Comput. Optim. Appl. 39 (2008) 183-218.

[24] J.-P. Raymond and F. Tröltzsch, Second order sufficient optimality conditions for nonlinear parabolic control problems with state constraints. Discrete Contin. Dyn. S. 6 (2000) 431-450.

[25] J.-P. Raymond and H. Zidani, Hamiltonian Pontryagin's principles for control problems governed by semilinear parabolic equations. Appl. Math. Optim. 39 (1999) 143-177.

[26] A. Rösch and F. Tröltzsch, Existence of regular Lagrange multipliers for a nonlinear elliptic optimal control problem with pointwise control-state constraints. SIAM J. Control Opt. 45 (2006) 548-564. 
[27] A. Rösch and F. Tröltzsch, Sufficient second-order optimality conditions for an elliptic optimal control problem with pointwise control-state constraints. SIAM J. Optim. 17 (2006) 776-794.

[28] A. Rösch and F. Tröltzsch, On regularity of solutions and Lagrange multipliers of optimal control problems for semilinear equations with mixed pointwise control-state constraints. SIAM J. Control Opt. 46 (2007) 1098-1115.

[29] A. Schiela, The control reduced interior point method. A function space oriented algorithmic approach. Ph.D. thesis, Freie Universität Berlin, Germany (2006).

[30] F. Tröltzsch and I. Yousept, A regularization method for the numerical solution of elliptic boundary control problems with pointwise state constraints. Comput. Optim. Appl. DOI: 10.1007/s10589-007-9114-0 (2008) online first. 